\title{
Tracing kinematical and physical asymmetries in the jet from DG Tauri B
}

\author{
L. Podio ${ }^{1}$, J. Eislöffel ${ }^{2}$, S. Melnikov ${ }^{3}$, K. W. Hodapp ${ }^{4}$, and F. Bacciotti ${ }^{5}$
}

1 Kapteyn Astronomical Institute, Landleven 12, 9747 AD Groningen, The Netherlands e-mail: podio@astro.rug.nl

2 Thüringer Landessternwarte Tautenburg, Sternwarte 5, 07778 Tautenburg, Germany e-mail: jochen@tls-tautenburg.de

3 Ulugh Beg Astronomical Institute, Astronomical str. 33, 700052 Tashkent, Uzbekistan e-mail: stas@astrin.uzsci.net

4 Institute for Astronomy, University of Hawaii, 640 N. Aohoku Place, Hilo, HI 96720, USA e-mail: hodapp@ifa.hawaii.edu

5 INAF - Osservatorio Astrofisico di Arcetri, Largo Enrico Fermi 5, 50125 Firenze, Italy e-mail: fran@arcetri.astro.it

Received 2 November 2010 / Accepted 6 December 2010

ABSTRACT

Context. Jets from young stars can be highly asymmetric and have multiple velocity components.

Aims. To clarify the origin of jet asymmetries and constrain the launch mechanism, we study as a test case the physical and kinematical structure of the prototypical asymmetric flow emitted by DG Tau B.

Methods. The analysis of deep, high spectral resolution observations taken with the KECK telescope allows us to infer the properties and the spatial distribution of the velocity components in the two jet lobes. From selected line ratios we derive the gas physical conditions (the electron and total density, $n_{\mathrm{e}}$ and $n_{\mathrm{H}}$, the ionisation fraction, $x_{\mathrm{e}}$, and the temperature, $T_{\mathrm{e}}$ ), as a function of the distance from the source and the gas velocity. The presence of dust grains in the jet is investigated by estimating the gas-phase abundance of calcium with respect to its solar value.

Results. The detected lines show broad velocity profiles at the base of the jet (up to $\sim 100 \mathrm{~km} \mathrm{~s}^{-1}$ ), where up to three velocity components are detected. At $5^{\prime \prime}$ from the source, however, only the denser and more excited high-velocity components survive and the lines are narrower $\left(\sim 10-30 \mathrm{~km} \mathrm{~s}^{-1}\right)$. The jet is strongly asymmetric in the velocity and in its physical structure. The red lobe, which is slower $\left(\sim 140 \mathrm{~km} \mathrm{~s}^{-1}\right)$ and more collimated (opening angle: $\left.\alpha \sim 3-4^{\circ}\right)$, presents low ionisation fractions $\left(x_{\mathrm{e}} \sim 0.1-0.4\right)$ and temperatures $\left(T_{\mathrm{e}}<5 \times 10^{3} \mathrm{~K}\right)$, while the total density is up to $\sim 2.5 \times 10^{4} \mathrm{~cm}^{-3}$. The blue lobe, faster $\left(\sim-320 \mathrm{~km} \mathrm{~s}^{-1}\right)$ and less collimated $\left(\alpha \sim 14^{\circ}\right)$, is also less dense $\left(n_{\mathrm{H}}<10^{4} \mathrm{~cm}^{-3}\right)$, but highly excited $\left(T_{\mathrm{e}}\right.$ up to $\sim 5 \times 10^{4} \mathrm{~K}$ and $x_{\mathrm{e}}$ up to 0.9$)$. The estimated mass-loss rate turns out to be similar in the two lobes $\left(\sim 6-8 \times 10^{-9} M_{\odot} \mathrm{yr}^{-1}\right)$, while the flux of the linear momentum is three times higher in the blue one $\left(\sim 2.5 \times 10^{-7} M_{\odot} \mathrm{yr}^{-1} \mathrm{~km} \mathrm{~s}^{-1}\right)$. Calcium is strongly depleted with respect to its solar abundance, indicating that the jet contains dust grains. The depletion is lower for higher velocities, which is consistent with dust destruction by shocks.

Conclusions. The similar mass-loss rate in the two lobes suggests that the ejection power is comparable on the two sides of the system, as expected from a magneto-centrifugal ejection mechanism, and that the observed asymmetries are caused by a different mass load and propagation properties in an inhomogeneous environment. The presence of dust grains implies that the jet is generated from a region of the disc extending beyond the dust sublimation radius.

Key words. ISM: jets and outflows - Herbig-Haro objects - dust, extinction - stars: formation - stars: individual: DG Tau B

\section{Introduction}

Jets from young stellar objects (YSO) play a key role in the starformation process, and a wealth of information can be derived by analysing their characteristic emission line spectra. The observed lines are collisionally excited in the shock waves that are generated by the interaction of the jet material with the interstellar medium or previously ejected matter and contain important information on the gas physics/kinematics. Different methods have been proposed to derive the physical conditions of the gas that propagates in the jets from the emission line ratios, such as the comparison with the predictions of a grid of shock models (e.g., Raga \& Böhm 1986; Hartigan et al. 1994), or, alternatively, spectral diagnostic techniques (e.g., Bacciotti \& Eislöffel 1999, hereafter referred to as the BE technique).

The application of these methods to analyse emission lines allows one to derive the jet structure on different scales, depending on the angular resolution of the observations: from parsec scales to hundreds of AU (Hartigan et al. 1994; Bacciotti \& Eislöffel 1999; Podio et al. 2006), and down to 15 AU from the emitting source with space or adaptive optics-assisted observations (Bacciotti et al. 2000; Lavalley-Fouquet et al. 2000; Woitas et al. 2002; Hartigan \& Morse 2007; Melnikov et al. 2009). In particular, the analysis of emission lines can shed light on the gas conditions at the base of the flow and on the mechanism that generates this flow. Proposed magneto hydrodynamical (MHD) models suggest that the wind is accelerated and collimated by magneto-centrifugal forces. It is not clear, however, where the jet originates: from the star itself (Sauty \& Tsinganos 1994), from the radius at which the stellar magnetosphere truncates the disc (X wind, Shu et al. 2000), or from an extended region of the disc (disc wind, Königl \& Pudritz 2000).

In addition to the uncertainty on the launch mechanism, other observed properties still lack a proper modelling. For example, 
velocity-resolved observations of the jets from $\mathrm{T}$ Tauri stars and younger Class 0/I protostars show that these can be highly asymmetric and can have different velocity components (Hamann 1994; Hirth et al. 1994, 1997; Davis et al. 2001; Garcia Lopez et al. 2008, 2010). Despite some attempts to explain these observational features by means of the existing MHD models (e.g., Pesenti et al. 2003; Ferreira et al. 2006), their origin is still unclear. A viable possibility to clarify these aspects is to investigate the variation of the jet physical properties in asymmetric jets and in different velocity channels, which has been attempted in recent works conducted at moderate and high spatial/spectral resolution (Lavalley-Fouquet et al. 2000; Woitas et al. 2002; Coffey et al. 2008; Garcia Lopez et al. 2008, 2010; Melnikov et al. 2009; Podio et al. 2009).

Another interesting issue, which remains not fully clarified, is the dust content of jets. Refractory species such as $\mathrm{Ca}, \mathrm{Ni}$, $\mathrm{Cr}$, and $\mathrm{Fe}$ are often locked onto dust grains, thus a strong depletion of their gas phase abundance is expected in the interstellar medium (ISM) (Savage \& Sembach 1996). A few studies have investigated the gas phase abundance of $\mathrm{Fe}$ and $\mathrm{Ni}$ in HH jets (Beck-Winchatz et al. 1994, 1996; Mouri \& Taniguchi 2000; Böhm \& Matt 2001; Nisini et al. 2002), and only recently the analysis has been extended to other refractory species such as Si, Ca, C, Cr, and Ti (Nisini et al. 2005, 2007; Cabrit et al. 2007; Podio et al. 2006, 2009; Garcia Lopez et al. 2008). These studies showed that the refractory species may be depleted by up to $90 \%$ in $\mathrm{HH}$ jets and molecular outflows, thus suggesting the presence of dust grains in the ejected material. This in turn can put constraints on the region of the disc from where jets originate. Indeed the stellar radiation destroys dust grains in the disc up to the so-called dust evaporation radius, $R_{\text {evp }}$. This is located between 0.03 and 1.5 AU from the star in T Tauri and Herbig Ae/Be stars depending on the stellar luminosity and the dust properties (Isella \& Natta 2005; Akeson et al. 2005; Eisner et al. 2007), but no direct observations and/or modelling of the inner disc structure are available for younger Class 0/I sources, which are still surrounded by circumstellar matter. In general, we expect that a "dusty" jet can only arise from a region in the disc extended beyond $R_{\text {evp }}$. The passage through a shock front, however, can destroy part of the dust grains that may be transported in the jet, which complicates the picture (e.g., May et al. 2000; Guillet et al. 2009). Therefore, accurate studies of the gas-phase abundance of refractory species are necessary to determine the dust content in the jet and to constrain the shock efficiency in destroying the dust grains.

To improve our understanding of the physics of $\mathrm{HH}$ jets from YSO, particularly their launch mechanism, we investigate the physical and kinematical structure of the $\mathrm{HH} 159$ jet emitted by the young source DG Tau B, focusing on the analysis of the velocity components detected at its base, the asymmetry between the two jet lobes, and, finally, the estimate of the dust content in the jet.

HH 159 is a bipolar jet, first detected by Mundt \& Fried (1983). The red lobe consists of a chain of bright knots extending to $\sim 55^{\prime \prime}$ from the source, while the blue lobe, fainter and less collimated, is detected only up to $\sim 10^{\prime \prime}$ from the source (Mundt et al. 1991; Eislöffel \& Mundt 1998). A large molecular outflow spatially coincident with the redshifted optical jet has been detected in the CO lines by Mitchell et al. (1994, 1997). The driving star, DG Tau B, has been classified as a Class I source based on its spectral energy distribution (Watson et al. 2004; Luhman et al. 2010) and at optical wavelengths it is obscured by circumstellar optically thick material detected in absorption through broadband imaging with the Hubble space telescope
(HST) (Stapelfeldt et al. 1997). The observed dark lane, elongated and perpendicular to the jet, indicates the presence of a circumstellar disc, confirmed by ${ }^{13} \mathrm{CO}$ millimeter observations (Padgett et al. 1999, 2000), and a flattened residual envelope. The source position has been determined from $3.5 \mathrm{~cm}$ VLA radio continuum observations by Rodriguez et al. (1995), who locate it within the observed dark lane. Further HST studies in the near-infrared indicated the presence of a bipolar reflection nebula, whose axis of symmetry is coincident with the axis of the optical jet (Padgett et al. 1999). The eastern lobe of the nebula, surrounding the blue jet lobe, is $V$-shaped, suggesting that the nebula traces the walls of the blueshifted outflow cavity. The western lobe is fainter and more collimated, and encompasses the redshifted optical jet and the CO outflow.

Previous spectroscopic (Mundt et al. 1987; Eislöffel \& Mundt 1998) and imaging (Mundt et al. 1991; Stapelfeldt et al. 1997; Padgett et al. 1999) studies highlighted a strong asymmetry between the two lobes in morphology, velocity, and degree of excitation. But these studies focused only on the $\mathrm{H} \alpha$ and [S II] emission lines, and also on the continuum emission in the optical and near-infrared bands. In contrast we here present for the first time very deep and high-spectral resolution spectroscopic observations of a large number of forbidden and permitted lines in the optical range from $5300 \AA$ to $8500 \AA$. The high spectral resolution coupled with long integration times highlights a very complex velocity structure with multiple velocity components evolving along the jet. The large number of detected lines has allowed us a detailed study of the gas physical conditions through the application of spectral diagnostic techniques to selected line ratios. This has provided rich information on the excitation and dynamics of the flow, as well as on the dust reprocessing. The comparison of our results with those obtained for other jets provides means of investigating the nature of the detected velocity components and the origin of asymmetric jets. This, in turn, can help understanding the generation of outflows and their role in young systems.

\section{Observations and data reduction}

High-resolution echelle spectra of the jet from DG Tau B were obtained with the HIRES spectrograph at the Keck I telescope (Vogt et al. 1994), located at the Keck Observatory on Mauna Kea, Hawaii, on 1998 February 23 and 24. The weather conditions were stable and photometric during the nights, providing a seeing of typically $0.7-0$. ' 8 . Four deep spectra of $2700 \mathrm{~s}$ integration time were obtained using a long slit decker of $28^{\prime \prime} \times 11^{\prime \prime} 15$, and a $2048 \times 2048$ CCD Tektronix detector with $2 \times$ binning in the spatial direction. This setting provided very high spectral resolution $\left(R \sim 34000\right.$, i.e. $\left.\sim 8.8 \mathrm{~km} \mathrm{~s}^{-1}\right)$, spectral sampling of $\sim 0.036-0.052 \AA \mathrm{pixel}^{-1}$, (corresponding to $\sim 2.15 \mathrm{~km} \mathrm{~s}^{-1}$ ), and a spatial scale of $\sim 0$ '. $^{\prime} 378$ pixel $^{-1}$.

The decker was aligned along the jet position angle (PA 295 , Eislöffel \& Mundt 1998). Because the decker is $28^{\prime \prime}$ long, i.e. shorter than the jet (Eislöffel \& Mundt 1998), to cover the full jet length the observations were carried out by centring the decker on the jet source, and then shifting it along the jet PA towards the south-east and north-west with respect to the source position, along the blue and the red lobe. In this way a length of $\sim 55^{\prime \prime}$ was covered along the spatial direction. The position angle tracking mode allowed us to keep the slit orientation at the chosen PA during the four exposures. By using the decker, 23 spectral orders overlapping along the spatial direction were obtained, covering a wavelength range from $5300 \AA$ to $8500 \AA$. 
The raw frames were reduced following standard recipes with the noao.imred.ccdred package in $\mathrm{IRAF}^{1}$, including overscan correction, bias and dark subtraction, flat-fielding, background subtraction, and wavelength calibration using ThoriumArgon arcs. The Laplacian Cosmic Ray Identification method ("lacos" method, van Dokkum 2001) was used to clean the images from cosmics.

Because of the characteristic jet emission line spectrum we are interested in short sections of the echelle spectra. Therefore, the latter were transformed into ordinary long-slit spectra centred around the detected lines. A flat-field frame obtained with a $28^{\prime \prime}$ long slit was used to calculate the tilt of the orders in the echelle images with respect to a normal long slit spectrum. Hence, the orders containing the emission lines were corrected for the estimated tilt. The same procedure was applied to the Thorium-Argon arcs frames.

Wavelength calibration for the selected spectral orders was performed by means of Thorium-Argon arcs, providing an accuracy of about $0.003 \AA$. The observed velocities were corrected for the velocity of DG Tau B with respect to the observer, hence the values of $V_{\text {rad }}$ showed in all the plots refer to the gas radial velocity with respect to the driving source. Because the central source is obscured, it is not possible to estimate the source radial velocity from photospheric absorption lines, e.g. Li I 16707. Thus, following Eislöffel \& Mundt (1998) we assume that DG Tau B has the same heliocentric radial velocity as the surrounding molecular gas $\left(\sim 16.3 \mathrm{~km} \mathrm{~s}^{-1}\right.$, Mitchell et al. 1994 ), which translates into a source velocity with respect to the observer of $\sim 46.4 \mathrm{~km} \mathrm{~s}^{-1}$.

Finally, the four frames centred on different positions along the jet PA were aligned and then combined by estimating the position of the brightest emission knots. Thus we obtain for each detected line a long slit spectrum covering $\sim 55^{\prime \prime}$ along the spatial direction and $\sim 310 \mathrm{~km} \mathrm{~s}^{-1}$ along the spectral one (see the position-velocity (PV) diagrams in Figs. 1 and 2 for selected lines). Note that in all the figures shown in this paper the distance from the source is positive along the blue lobe and negative along the red one.

At the end of the data reduction process, telluric features overlapping with the jet emission lines and background caused by the overlap of adjacent orders were fitted by a polynomial surface and then subtracted. The extremely bright [OI] $\lambda 6300$ telluric line could not be properly subtracted, thus the $\left[\mathrm{O}_{\mathrm{I}}\right] \lambda 6300$ line $\mathrm{PV}$ shows a $\sim 20 \mathrm{~km} \mathrm{~s}^{-1}$ cut in the blue lobe centred at $\sim-46.4 \mathrm{~km} \mathrm{~s}^{-1}$ (see Fig. 1). This cut is also clearly visible in the PV diagrams of the derived line ratios and of the jet physical properties (see Figs. 5 and 6).

Note that the spectra are not flux-calibrated because no standard-star observations are available. Thus, the intensity of the lines in Figs. 1 and 2 is in arbitrary units with respect to the background noise, $\sigma$.

\section{Results}

The deep KECK/HIRES spectra (see Figs. 1 and 2) allowed us to analyse the emission of the $\mathrm{HH} 159$ jet up to $\sim 40^{\prime \prime}$ in the bright red lobe (knots A1, A3, A2, B2, C, and D, following the nomenclature by Eislöffel \& Mundt 1998) and $\sim 10^{\prime \prime}$ in the fainter blue lobe (knot F1, F2, and G). Besides the [S II] $\lambda \lambda 6716$, 6731 and $\mathrm{H} \alpha \lambda 6563$ lines, which have been previously observed

\footnotetext{
1 IRAF is distributed by National Optical Astronomy Observatories, which is operated by the Association of Universities for Research in Astronomy, Inc. under contract with the National Science Foundation.
}

by Mundt et al. (1987, 1991) and Eislöffel \& Mundt (1998), we detected strong and broad emission in [O I] $\lambda \lambda 6300,6364$, and in $\left[\mathrm{N}_{\text {II }}\right] \lambda \lambda 6548,6583$ (see Fig. 1). The jet is emitting also in a number of fainter and narrower emission lines, such as $[\mathrm{Fe}$ II $] \lambda 5159$, $\left[\mathrm{N}_{\mathrm{I}}\right] \lambda \lambda 5198,5200,[\mathrm{Ca} \mathrm{II}] \lambda 7291$, and $[\mathrm{Ni} \mathrm{II}] \lambda 7378$, detected up to $\sim 20^{\prime \prime}$ (knot C) (see Fig. 2). Finally, very faint emission in the $\mathrm{Na}$ I D lines at $\lambda \lambda 5890,5896$ is also detected at a $\sim 6 \sigma$ level in knots A1, A3. Because the ionisation energy of $\mathrm{Na}$ I is very low, i.e. $\sim 5.1 \mathrm{eV}$, the $\mathrm{Na}$ I $\mathrm{D}$ lines are rarely observed in $\mathrm{HH}$ objects (see, e.g., HH 1 spectra by Solf et al. 1988). The detection of these lines suggests that the excitation conditions are quite low in the red lobe. This idea is confirmed by our analysis of the gas physical conditions (see Sect. 3.2).

\subsection{Jet kinematics: asymmetries and velocity components}

The PV diagrams of selected emission lines in Fig. 1 show very broad (up to $\sim 200 \mathrm{~km} \mathrm{~s}^{-1} \mathrm{FWZI}$ ) and complex (up to three velocity components) velocity profiles in the first few arcseconds from the source. Thanks to the high spectral resolution of the data $\left(\sim 8.8 \mathrm{~km} \mathrm{~s}^{-1}\right)$, we can resolve and analyse each velocity component in the jet separately. To derive the kinematical properties we extracted the jet spectral profile in the two lobes by spatially integrating the $\left[\mathrm{S}_{\mathrm{II}}\right] \lambda 6731$ and $\mathrm{H} \alpha$ emission lines over the seeing full width at half maximum $\left(F W H M_{\text {seeing }} \sim 0.0^{\prime} 7-0 .{ }^{\prime} 8\right.$, i.e. $\sim 2$ pixels) at different distances along the jet length. Figure 3 shows the variation of the $\left[\mathrm{S}_{\mathrm{II}}\right] \lambda 6731$ and $\mathrm{H} \alpha$ velocity profile in the red and the blue lobe up to $\sim 6^{\prime \prime}$ from the source. The red lobe clearly shows a low- and a high-velocity component (LVC and HVC), while in the blue lobe we detect three velocity components, at low, moderate, and high velocities (LVC, MVC, and $\mathrm{HVC}$, respectively). Interestingly, in both lobes the lowand moderate-velocity components fade away at $\sim 4-5^{\prime \prime}$ from the source, while the high-velocity components survive farther out. This trend has been observed on a large sample of T Tauri stars, but on much smaller scales, i.e. between a few tens and $200 \mathrm{AU}$ from the source, by, e.g., Hirth et al. (1997) and Pyo et al. (2003), and on similar or even larger scales at the base of $\mathrm{HH}$ jets from Class 0/I sources (Garcia Lopez et al. 2008, 2010).

By deblending and Gaussian fitting the spectral profiles shown in Fig. 3 we obtain the gas radial velocity with respect to DG Tau B, $V_{\text {rad }}$, and the spectral width of each velocity component in the jet, $\Delta V$, measured as the observed line full width at half maximum $\left(F W H M_{\text {obs }}\right)$ corrected for the instrumental width $\left(F W H M_{\text {inst }} \sim 8.8 \mathrm{~km} \mathrm{~s}^{-1}\right)$. This is done by assuming a Gaussian profile for the instrumental and the emission-line profile (i.e. $\left.\Delta V=\left(F W H M_{\text {obs }}^{2}-F W H M_{\text {inst }}{ }^{2}\right)^{1 / 2}\right)$. The error on the obtained determination of $V_{\mathrm{rad}}$ and $\Delta V$ varies between 1$4 \mathrm{~km} \mathrm{~s}^{-1}$. The parameters derived by fitting the [S II] $\lambda 6731$ and the $\mathrm{H} \alpha \lambda 6563$ line profiles are plotted in Fig. 4 as a function of distance from the source.

The [S II] $\lambda 6731$ line profiles in Fig. 3 show very well-defined velocity components. In the red lobe the HVC radial velocity is decreasing in the first $14^{\prime \prime}$ from the source from $\sim 60 \mathrm{~km} \mathrm{~s}^{-1}$ in the innermost knots $\mathrm{A} 1, \mathrm{~A} 3$ down to $\sim 47 \mathrm{~km} \mathrm{~s}^{-1}$ in knots B2. Then $V_{\text {rad }}$ is increasing again, moving towards the outer knots $\mathrm{C}\left(V_{\mathrm{rad}} \sim 50-55 \mathrm{~km} \mathrm{~s}^{-1}\right)$ and $\mathrm{D}\left(V_{\mathrm{rad}} \sim 55-60 \mathrm{~km} \mathrm{~s}^{-1}\right)$. This trend, clearly visible also in the line PV diagrams (see Figs. 1 and 2), can be explained if the jet direction is changing because of precession or interaction with the ambient medium. Observational evidence of precessing jets has been already reported by, e.g., Giannini et al. (2005), Hodapp et al. (2005), and Caratti o Garatti et al. (2008). The LVC peak velocity instead varies between 25 and $42 \mathrm{~km} \mathrm{~s}^{-1}$. In the blue lobe much higher 


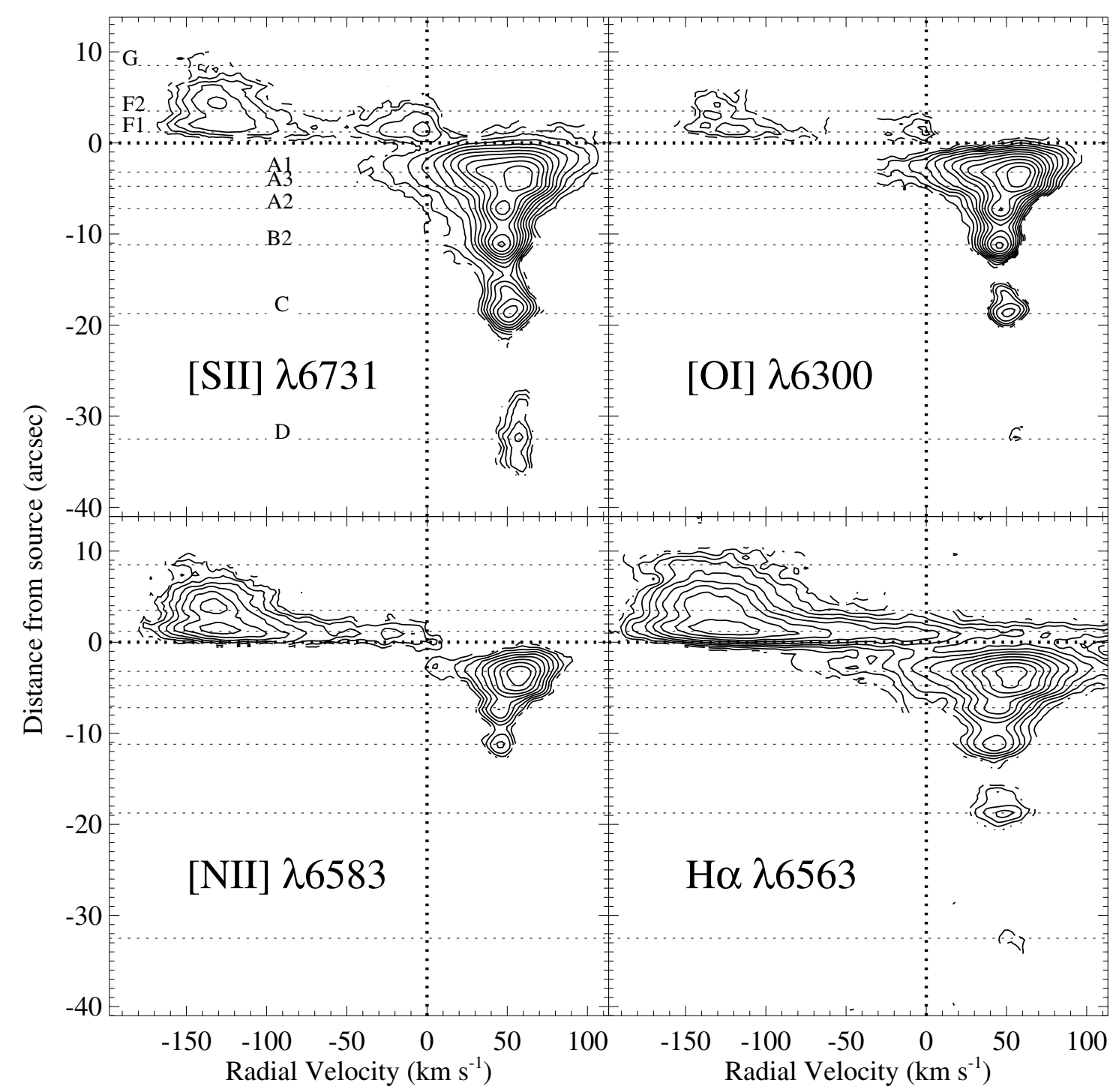

Fig. 1. Position-velocity diagrams of the jet from DG Tau B in the strong optical emission lines [S II $] \lambda 6731$, [O I $] \lambda 6300,\left[\mathrm{~N}_{\text {II }}\right] \lambda 6583$, and $\mathrm{H}_{\alpha \lambda}{ }_{6563}$ (angular resolution: $F W H M_{\text {seeing }} \sim 0.8^{\prime \prime}$, spectral resolution: $F W H M_{\text {inst }} \sim 8.8 \mathrm{~km} \mathrm{~s}^{-1}$ ). Contours are equally spaced in logarithmic scale. The first

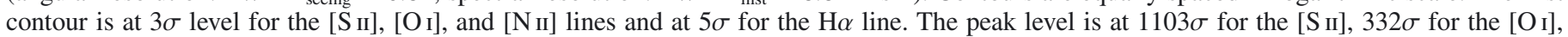
$115 \sigma$ for the [N $\left.\mathrm{N}_{\mathrm{II}}\right]$, and $131 \sigma$ for the $\mathrm{H} \alpha$ line. The vertical and horizontal dotted lines indicate the zero radial velocity with respect to the star and the spatial position of the source. The dashed horizontal lines indicate the position of the detected knots.

radial velocities are measured in the $\operatorname{HVC}\left(\sim 120-135 \mathrm{~km} \mathrm{~s}^{-1}\right)$, while $V_{\mathrm{rad}} \sim 20-30 \mathrm{~km} \mathrm{~s}^{-1}$ in the MVC, and a few $\mathrm{km} \mathrm{s}^{-1}$ in the LVC. Given the jet inclination angle with respect to the line of sight of $\sim 65^{\circ}$ estimated through proper motions measurements (Eislöffel \& Mundt 1998), the bulk of the gas is moving with a velocity $V_{\text {jet }} \sim 140 \mathrm{~km} \mathrm{~s}^{-1}$ in the red lobe, and more than twice as high $\left(V_{\text {jet }} \sim-320 \mathrm{~km} \mathrm{~s}^{-1}\right)$ in the blue one. These values agree with those estimated by Eislöffel \& Mundt (1998). The width, $\Delta V$, of the high-velocity component is decreasing with distance from the source in both lobes. In the blue lobe we measure $\Delta V \sim 60 \mathrm{~km} \mathrm{~s}^{-1}$ at $\sim 1^{\prime \prime}$, decreasing to $\sim 30 \mathrm{~km} \mathrm{~s}^{-1}$ at $\sim 6^{\prime \prime}$. In the red lobe $\Delta V$ decreases more steeply from $\sim 40-60 \mathrm{~km} \mathrm{~s}^{-1}$ within $1^{\prime \prime}$ from the source down to $\sim 15 \mathrm{~km} \mathrm{~s}^{-1}$ at $\sim 3^{\prime \prime}$, and values between $6-20 \mathrm{~km} \mathrm{~s}^{-1}$ for larger distances.

The $\mathrm{H} \alpha \lambda 6563$ line profiles are much broader than the [S II] profiles (FWZI up to $\sim 200 \mathrm{~km} \mathrm{~s}^{-1}$ ) and the emission is very diffuse in this line, making it difficult to separate the different velocity components. Thus we only report the values of $V_{\mathrm{rad}}$ and
$\Delta V$ estimated for the HVC of the red lobe. Figure 4 shows that $V_{\text {rad }}$ has the same trend as in the $[\mathrm{S}$ II] lines, with only slightly lower values. The line width is much larger, though $(\Delta V$ varies between $\sim 35$ and $\sim 100 \mathrm{~km} \mathrm{~s}^{-1}$ ). A broader $\mathrm{H} \alpha$ line profile was also noted by Eislöffel \& Mundt (1998).

The large velocity dispersion measured within a few arcseconds from the source and, in particular, in the blue lobe, suggests that stronger shocks are present at the jet base, and in the blue lobe. As we will show in the next section, this reflects in higher excitation conditions in the blue lobe, that is the jet is highly asymmetric not only in velocity, but also in its physical structure.

\subsection{Jet physical structure: density and excitation}

The position-velocity diagrams shown in Fig. 1 suggest a strong asymmetry of the gas excitation conditions in the two jet lobes. Low excitation lines, such as $\left[\mathrm{S}_{\mathrm{II}}\right] \lambda 6731$ and $\left[\mathrm{O}_{\mathrm{I}}\right] \lambda 6300$, are 


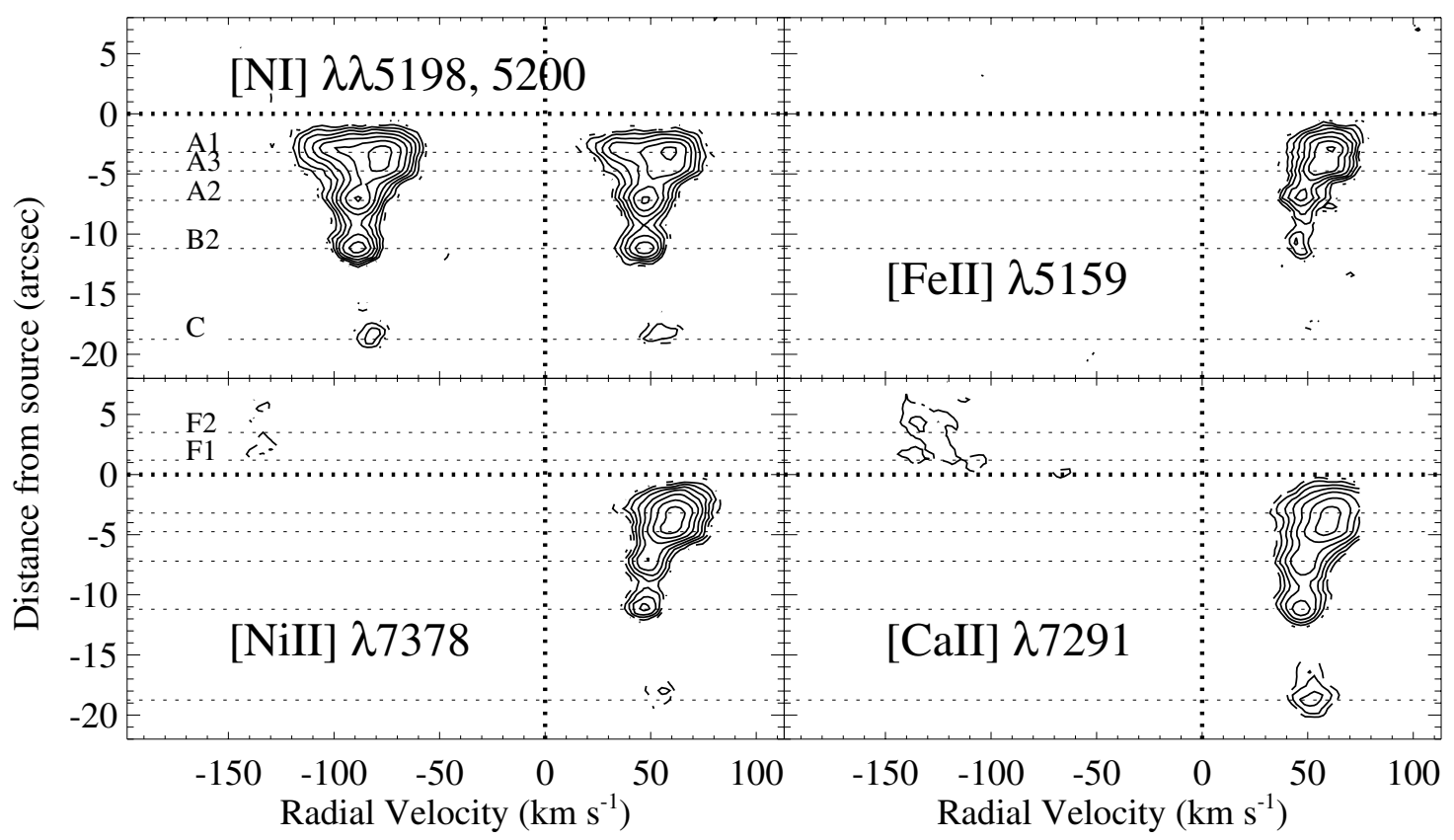

Fig. 2. Position-velocity diagrams of the jet from DG Tau B in the $\left[\mathrm{N}_{\mathrm{I}}\right] \lambda \lambda 5198,5200,[\mathrm{Fe}$ II $] \lambda 5159,[\mathrm{Ni}$ I $] \lambda 7378$, $[\mathrm{Ca}$ II $] \lambda 7291$ emission lines. Note that the velocity scale in the first panel is given with regard to the $\left[\mathrm{N}_{\mathrm{I}}\right] \lambda 5200$ line, while the scale for the $\left[\mathrm{N}_{\mathrm{I}}\right] \lambda \lambda 5198$ plotted in the same panel is shifted by $-136 \mathrm{~km} \mathrm{~s}^{-1}$ in velocity. Contours are equally spaced in logarithmic scale. The first contour is at $2 \sigma$ level. The peak level is at $37 \sigma$ for the $\left[\mathrm{N}_{\mathrm{I}}\right], 24 \sigma$ for the $[\mathrm{Fe}$ II], $37 \sigma$ for the $[\mathrm{Ni}$ II], and $80 \sigma$ for the [Ca II] line. The vertical and horizontal dotted lines indicate the zero radial velocity with respect to the star and the spatial position of the source. The dashed horizontal lines indicate the position of the detected knots.

much fainter in the blue lobe than in the red one, while higher excitation lines, such as $[\mathrm{N}$ II] $\lambda 6583$ and $\mathrm{H} \alpha \lambda 6563$, are equally bright in the two lobes (see also the top panel of Fig. 7). This suggests that the gas in the blue lobe is not only moving at higher velocity, as inferred in the previous section, but is also more excited with respect to the gas in the red lobe.

In order to examine the jet physical conditions we compute selected line ratios that are known to be good tracers of the gas excitation (see Fig. 5). [S II] $]$ 6731/6716 probes the electron density, $n_{\mathrm{e}}$, increasing up until $n_{\mathrm{e}}$ reaches a critical density of $2.5 \times 10^{4} \mathrm{~cm}^{-3}$. On the other hand, $[\mathrm{N}$ II $] \lambda(6548+6583) /[\mathrm{OI}] \lambda(6300+6364)$ (hereafter $\left.[\mathrm{N} \mathrm{II}] /\left[\mathrm{O}_{\mathrm{I}}\right]\right)$ and $\left[\mathrm{O}_{\mathrm{I}}\right] \lambda(6300+6364) /\left[\mathrm{S}_{\mathrm{II}}\right] \lambda(6716+6731)$ (hereafter [O I $] /$ [S II]) strongly depend on the gas ionisation fraction and temperature (Bacciotti \& Eislöffel 1999). In particular, the [N II]/[O I] line ratio is increasing with $x_{\mathrm{e}}$ while $\left[\mathrm{O}_{\mathrm{I}}\right] /[\mathrm{S} \mathrm{II}]$ increases with $T_{\mathrm{e}}$ and the density. Finally, the $[\mathrm{S} \mathrm{II}] \lambda(6716+6731) / \mathrm{H} \alpha \lambda 6563$ line ratio (hereafter $[\mathrm{S} \mathrm{II}] / \mathrm{H} \alpha$ ) is an indicator of the excitation level (see, e.g., the predictions from shock models by Hartigan et al. 1994). In agreement with Mundt et al. (1987, 1991), we found that $[\mathrm{S}$ II] $/ \mathrm{H} \alpha$ can be as high as $\sim 20$ in the red lobe and very low $(\sim 0.2-1)$ in the blue one. This confirms that the redshifted jet is generally of low excitation, while the gas in the blue lobe is highly excited.

By applying the so-called BE technique, described in Bacciotti \& Eislöffel (1999), to the computed [S II] $66731 / 6716$, $\left[\mathrm{N}_{\mathrm{II}}\right] /\left[\mathrm{O}_{\mathrm{I}}\right]$, and $\left[\mathrm{O}_{\mathrm{I}}\right] /\left[\mathrm{S}_{\mathrm{II}}\right]$ ratios pixel by pixel we obtain position-velocity maps of the parameters characterizing the gas conditions, i.e. $n_{\mathrm{e}}, x_{\mathrm{e}}, T_{\mathrm{e}}$, and $n_{\mathrm{H}}$ (Fig. 6). The method relies on the fact that the observed forbidden lines are collisionally excited and that, in low-excitation conditions, and provided that no strong sources of ionising photons are present, the ionisation fraction of hydrogen is tightly related to those of nitrogen and oxygen via charge exchange processes.
Bacciotti \& Eislöffel (1999) and, later, Nisini et al. (2005), verified that the lines used in the technique are emitted in a region of similar temperature, density, and ionisation fraction. Hartigan $\&$ Morse (2007) further confirmed the validity of the method by applying spectral diagnostic techniques to infer the physical properties of the HH 30 jet. With this technique one can retrieve the gas physical conditions comparing the observed line ratios with the ones computed for a grid of values of $n_{\mathrm{e}}, x_{\mathrm{e}}$, and $T_{\mathrm{e}}$. The errors affecting the estimated parameters are due mainly to the errors on the measured line fluxes, i.e. on the signal-to-noise (see Podio et al. 2006, 2009, for a discussion on the computation of the errors). Note that the diagnostic technique uses ratios between different species and, thus, the obtained $x_{\mathrm{e}}$ and $T_{\mathrm{e}}$ may depend on the chosen set of elemental abundances. However, as stressed in Podio et al. (2006), the relative variations of the parameters (i.e. in different knots and/or velocity components) do not depend on the choice of an abundance set (see Fig. 1 of Podio et al. 2006) which, therefore, can be thought of as a model parameter. Moreover, the most recent determinations by Asplund et al. (2005) (solar) and Esteban et al. (2004) (Orion) agree well and produce diagnostic results that differ by not more than $15 \%$, which in most cases is less than the uncertainty derived by measurements errors. Since the inferred physical parameters are used also to determine the calcium gas-phase abundance with respect to solar (see Sect. 4.2), we adopt the solar abundances estimated by Asplund et al. (2005), as in Podio et al. (2009). We did not apply a correction for extinction to the spectra, but we note that, although the source is highly extincted by the surrounding optically thick disc $\left(A_{\mathrm{V}} \sim 6.5 \mathrm{mag}\right.$ or higher, Jones \& Cohen 1986; Watson et al. 2004), the Balmer decrement measured in the inner knots $\mathrm{A}$ and $\mathrm{F}(\mathrm{H} \alpha / \mathrm{H} \beta \sim 6-7$, Jones $\&$ Cohen 1986) indicates that $A_{\mathrm{V}}$ is always $<1$ along the jet. (According to shock models $\mathrm{H} \alpha / \mathrm{H} \beta$ may vary between $\sim 6-7$ in low-velocity shocks $\left(V_{\mathrm{s}} \sim 20 \mathrm{~km} \mathrm{~s}^{-1}\right)$ down to a minimum 

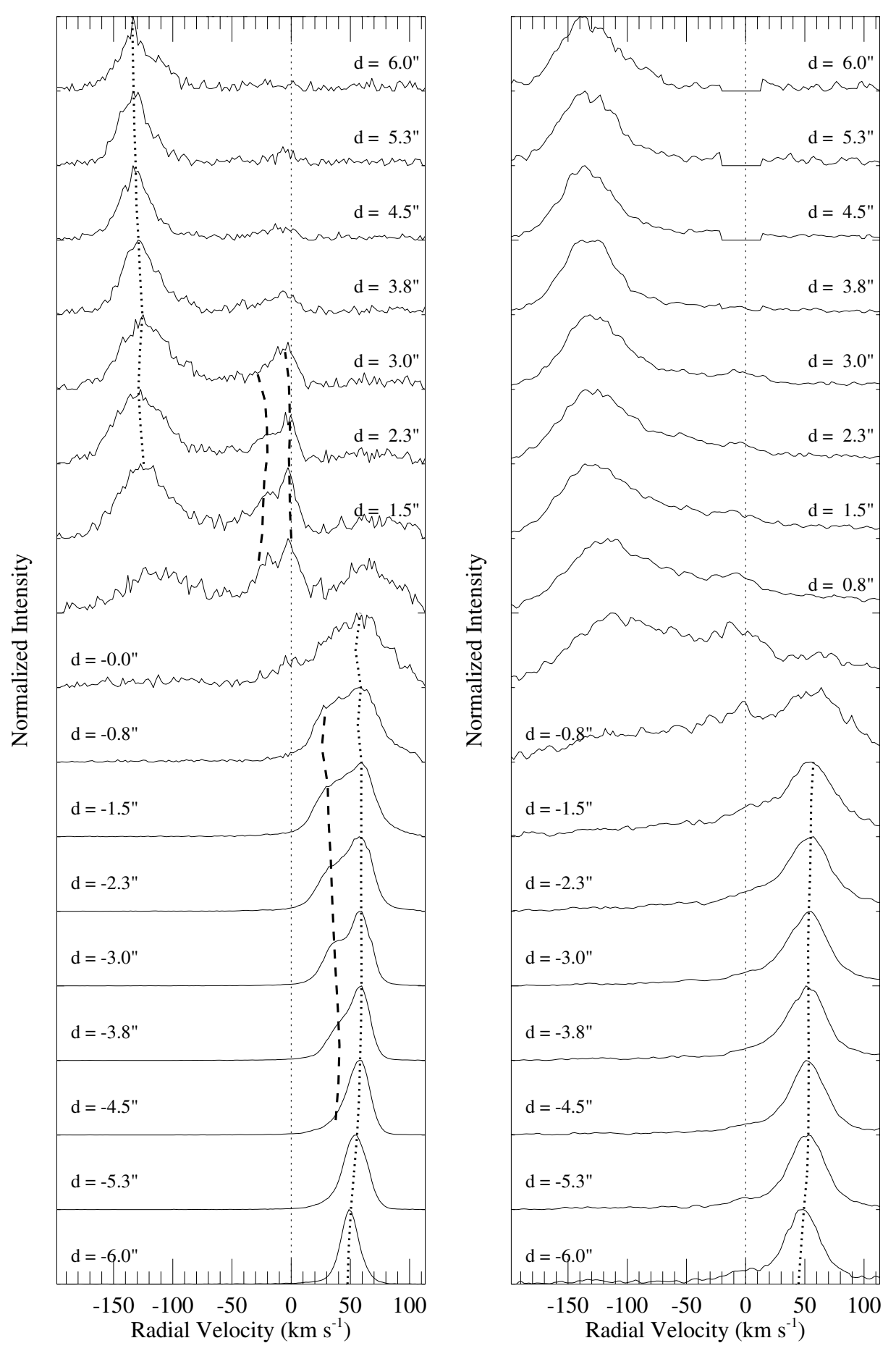

Fig. 3. Normalized [S II] $\lambda 6731$ (left panel) and $\mathrm{H} \alpha \lambda 6563$ (right panel) velocity profiles along the first $6^{\prime \prime}$ of the blue and the red lobe, obtained from the line PV diagrams in Fig. 1 by spatially integrating the line spectral profiles over the seeing $F W H M\left(\sim 0.8^{\prime \prime}\right.$, i.e. $\sim 2$ pixels). The label at each profile indicates the distance from the star. The vertical dotted line indicates the zero radial velocity with respect to the star. The dotted and dashed thick lines indicate the velocity peak of the high and the low (and moderate) velocity components, respectively. Note that beyond $\sim 5^{\prime \prime}$, only the high-velocity component survives in both lobes.

value of $\sim 3$ in high-velocity shocks $\left(V_{\mathrm{s}} \sim 100 \mathrm{~km} \mathrm{~s}^{-1}\right)$, Hartigan et al. 1994). On the other hand, Bacciotti \& Eislöffel (1999) show that because the lines used in this diagnostic are very close in wavelength, errors introduced by not correcting for extinction are at most $8 \%-10 \%$ for the ionisation fraction and $15 \%$ for the temperature, as long as the visual extinction is lower than $\sim 3$. To check the obtained $x_{\mathrm{e}}$ values we compare the observed $\left[\mathrm{N}_{\mathrm{II}}\right] \lambda(6548+6583) /\left[\mathrm{N}_{\mathrm{I}}\right] \lambda(5198+5200)$ with those computed using the inferred parameters. We selected the pixels with high $S / N$ because the $\left[\mathrm{N}_{\mathrm{I}}\right]$ lines are much fainter than the lines used in the diagnostics. For $S / N>20$ we find that observed and computed ratios agree within $20 \%-30 \%$, i.e. within the error.
The PV diagrams in Fig. 6 show the variation of the electron and total density, $n_{\mathrm{e}}$ and $n_{\mathrm{H}}$, the ionisation fraction, $x_{\mathrm{e}}$, and the temperature, $T_{\mathrm{e}}$, along the two jet lobes and as a function of the gas velocity. The PV diagrams indicate that the jet is denser and more excited close to the source and at high velocities in both lobes. The diagnostic confirms the strong asymmetry between the two lobes, the blue one being more excited, with values of the ionisation fraction going up to $\sim 0.9$ and temperatures up to $5 \times 10^{4} \mathrm{~K}$.

To have a clear view of the variation of the gas physical conditions along the two jet lobes and in the different velocity components we computed at each position along the jet the median of $n_{\mathrm{e}}, x_{\mathrm{e}}, T_{\mathrm{e}}$, and $n_{\mathrm{H}}$ on a $\sim 20 \mathrm{~km} \mathrm{~s}^{-1}$ velocity interval centred 
L. Podio et al.: Kinematical/physical asymmetries in the jet from DG Tau B

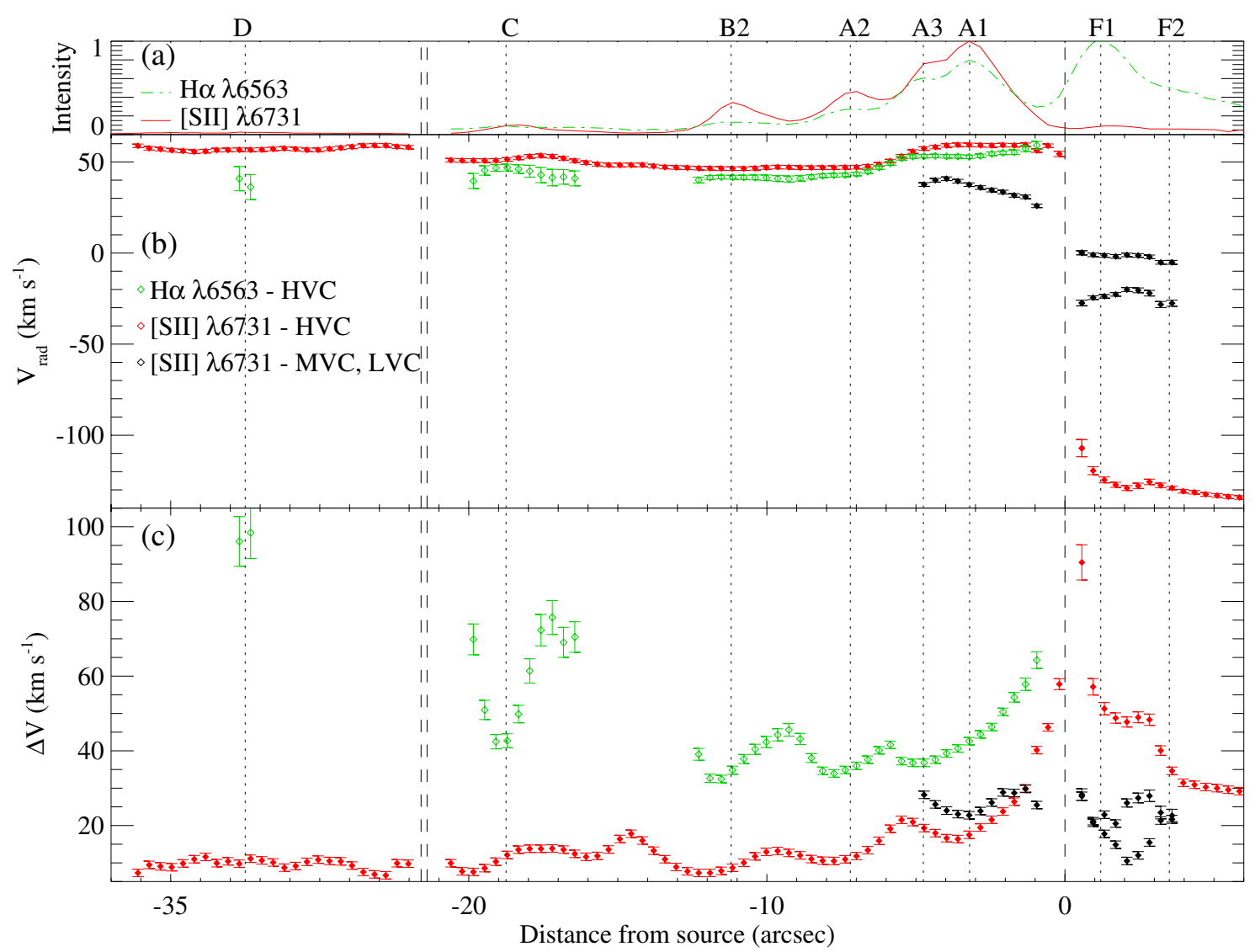

Fig. 4. Jet kinematical properties as a function of the distance from the source. From top to bottom: a) intensity profiles of the [S II] and the H $\alpha$ lines integrated over their velocity profile and normalized to their peak intensity (red solid and green dotted-dashed line, respectively); b), c) peak radial velocity, $V_{\text {rad }}$, and full width at half maximum, $\Delta V$, of the different velocity components in the jet as inferred from Gaussian deblending of the [S II] $\lambda 6731$ (HVC: red points, MVC, LVC: black points) and the $\mathrm{H} \alpha \lambda 6563$ (HVC: green points) line profiles shown in Fig. 3.

Table 1. Kinematical, physical, and dynamical properties of the jet from DG Tau B in the high- and the low-velocity components (HVC and LVC, respectively) averaged over the first $5^{\prime \prime}$ of the red and the blue lobe.

\begin{tabular}{cccccccccc}
\hline \hline Lobe & VC & $\begin{array}{c}V_{\text {rad }} \\
\mathrm{km} \mathrm{s}^{-1}\end{array}$ & $\begin{array}{c}\Delta V \\
\mathrm{~km} \mathrm{~s}^{-1}\end{array}$ & $\begin{array}{c}n_{\mathrm{e}} \\
10^{3} \mathrm{~cm}^{-3}\end{array}$ & $x_{\mathrm{e}}$ & $\begin{array}{c}T_{\mathrm{e}} \\
10^{4} \mathrm{~K}\end{array}$ & $\begin{array}{c}n_{\mathrm{H}} \\
10^{4} \mathrm{~cm}^{-3}\end{array}$ & $\begin{array}{c}\dot{M}_{\text {jet }} \\
10^{-9} M_{\odot} \mathrm{yr}^{-1}\end{array}$ & $\begin{array}{c}\dot{P}_{\text {jet }} \\
10^{-6} M_{\odot} \mathrm{yr}^{-1} \mathrm{~km} \mathrm{~s}^{-1}\end{array}$ \\
\hline Red & HVC & $58(54)$ & $27(46)$ & 3.3 & 0.22 & 0.45 & 1.49 & 4.2 & 0.6 \\
& LVC & 35 & 26 & 1.9 & 0.17 & 0.40 & 1.29 & 2.2 & 0.2 \\
Blue & HVC & -126 & 47 & 3.6 & 0.88 & 2.46 & 0.41 & 8.2 & 2.5 \\
& LVC & -2 & 18 & 1.1 & 0.41 & 0.74 & 0.29 & 0.09 & $7 \times 10^{-4}$ \\
\hline
\end{tabular}

Notes. The values of $V_{\text {rad }}$ and $\Delta V$ are derived from Gaussian fits to the [S II] $\lambda 6731$ line profiles and to the $\mathrm{H} \alpha \lambda 6563$ (in parentheses).

on the peak velocity of the low- and high-velocity components. The obtained values are shown in Fig. 7, while their average over the first $5^{\prime \prime}$ where the HVC and LVC are detected, is reported in Table 1. The contribution of the MVC, only barely visible in the blue lobe, is not significant and it is not reported in the figure and in the table. The error bars are the median of the errors on the estimated gas physical parameters over the selected $\sim 20 \mathrm{~km} \mathrm{~s}^{-1}$ interval. Note that these are much larger in the blue lobe where the signal-to-noise is much lower. The electron density $n_{\mathrm{e}}$ has a symmetrical trend on the first few arcseconds of the two lobes, decreasing from values of $\sim 6-8 \times 10^{3} \mathrm{~cm}^{-3}$ close to the source to $\sim 1 \times 10^{3} \mathrm{~cm}^{-3}$ at $\sim 6^{\prime \prime}$ in the HVC, and from $\sim 3 \times 10^{3} \mathrm{~cm}^{-3}$ to $\sim 500 \mathrm{~cm}^{-3}$ in the LVC. It is then even lower in knots $\mathrm{C}$ and $\mathrm{D}$ of the red lobe, where it reaches values of $\sim 200 \mathrm{~cm}^{-3}$. The excitation conditions, however, are dramatically different in the two lobes. The gas in the blue lobe is very hot $\left(T_{\mathrm{e}}(\mathrm{HVC}) \sim 1-5 \times 10^{4} \mathrm{~K}, T_{\mathrm{e}}(\mathrm{LVC}) \sim 0.6-1 \times 10^{4} \mathrm{~K}\right)$ and highly ionised $\left(x_{\mathrm{e}}(\mathrm{HVC}) \sim 0.7-0.8\right.$ and $\left.x_{\mathrm{e}}(\mathrm{LVC}) \sim 0.3-0.5\right)$. On the contrary, in the red lobe the temperature is lower $\left(T_{\mathrm{e}} \sim 3-\right.$ $\left.5 \times 10^{3} \mathrm{~K}\right)$ as well as the degree of ionisation $\left(x_{\mathrm{e}} \sim 0.1-0.3\right)$. Finally, the total hydrogen density is higher in the red lobe, where it decreases from $\sim 2-2.5 \times 10^{4} \mathrm{~cm}^{-3}$ in the first $1.5^{\prime \prime}$ down to $\sim 5 \times 10^{3}, \sim 3 \times 10^{3}$, and $\sim 1 \times 10^{3} \mathrm{~cm}^{-3}$ in knots A2, B2 and C, respectively; $n_{\mathrm{H}}$ is much lower in the blue lobe, decreasing from $\sim 1 \times 10^{4} \mathrm{~cm}^{-3}$ in knot F1 to $\sim 1 \times 10^{3} \mathrm{~cm}^{-3}$ in knot F2.

That electron and total density peak close to the source, where the velocity dispersion is higher, suggests that stronger shocks are occurring at the jet base that compress and heat the flow.

\section{Discussion}

In this section we derive from the inferred jet kinematical and physical properties crucial information on the dynamics of the 

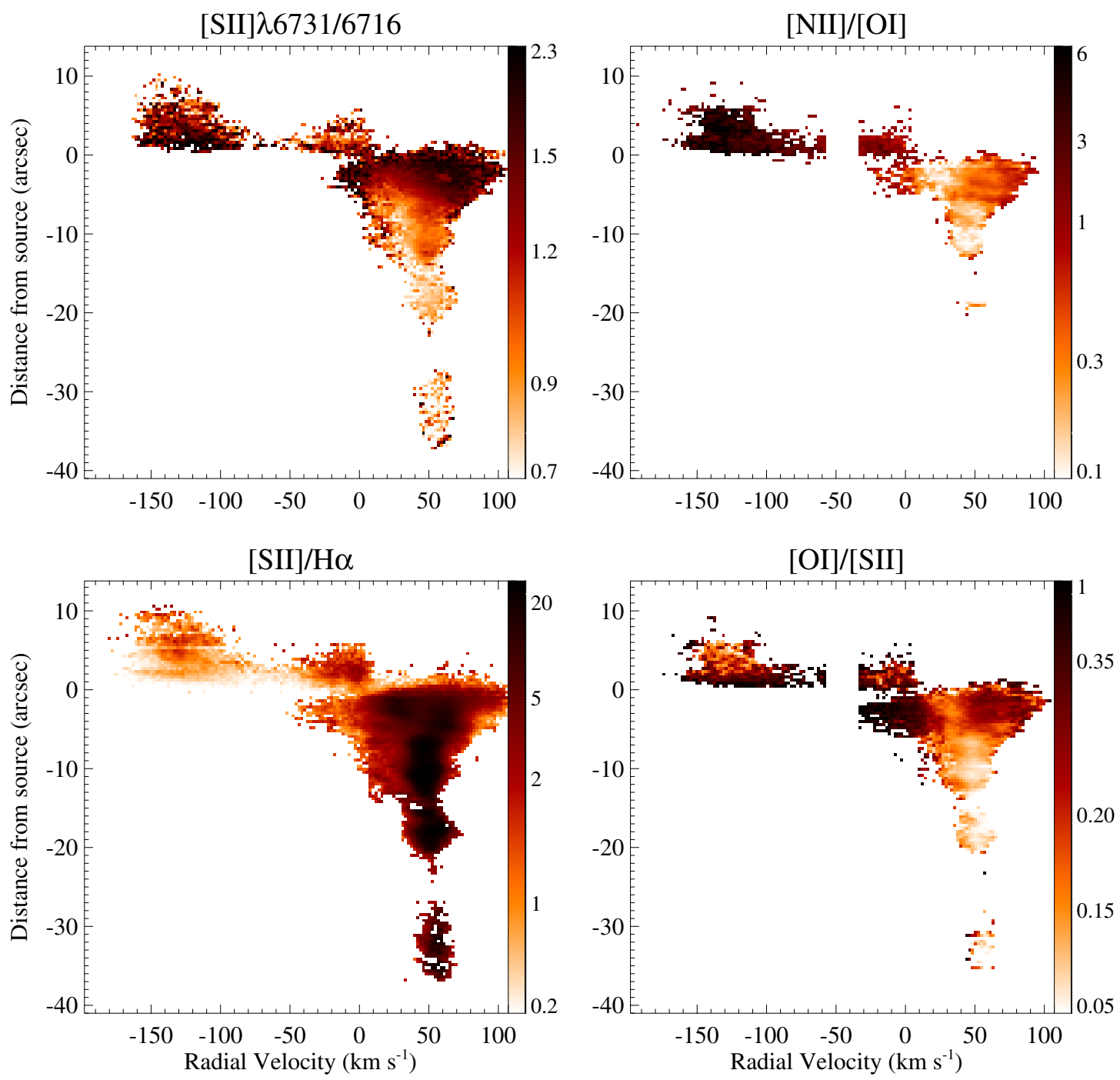

Fig. 5. Position-velocity diagrams of the line ratios used to infer the gas physical conditions in the jet: the [S II] $\lambda 6731 / 6716$ ratio increases with electron density, $n_{\mathrm{e}}$ (top left); the $\left[\mathrm{N}_{\mathrm{II}}\right] /\left[\mathrm{O}_{\mathrm{I}}\right]$ ratio mainly depends on the ionisation fraction, $x_{\mathrm{e}}$, and increases for increasing $x_{\mathrm{e}}$ (top right); the $[\mathrm{S} \mathrm{II}] / \mathrm{H} \alpha$ ratio is decreasing for increasing excitation conditions (bottom left); the $\left[\mathrm{O}_{\mathrm{I}}\right] /\left[\mathrm{S}_{\mathrm{II}}\right]$ ratio depends on all the parameters, but is mainly increasing with increasing temperature, $T_{\mathrm{e}}$, and density $n_{\mathrm{e}}$ (bottom right). To better visualize the variations of the line ratios a histogram equivalent colour scale is used.

outflows, that is the mass and momentum transported by the two jet lobes in their different velocity components. We then investigate the depletion of refractory species in gas phase, and finally we discuss the jet asymmetry and the kinematical/physical structure of the detected velocity components comparing our results with those obtained for other jets from $\mathrm{T}$ Tauri and younger Class 0/I stars.

\subsection{Jet dynamics: mass and momentum flux rates}

The mass and momentum flux transported by the jet, $\dot{M}_{\text {jet }}$ and $\dot{P}_{\text {jet}}$, are fundamental parameters to understand the dynamical relationship between the jet and its environment, and to test the validity of the magneto hydro-dynamical models proposed to explain the jet launch. For example, the models predict that the ratio between the rate of mass ejected into the jet $\left(\dot{M}_{\text {jet }}\right)$ and accreted from the disc onto the star $\left(\dot{M}_{\text {acc }}\right)$ is fixed $\left(\dot{M}_{\text {jet }} / \dot{M}_{\text {acc }} \sim 0.01-0.1\right.$, Shu et al. 2000; Königl \& Pudritz 2000).

As shown in previous studies (Hartigan et al. 1994; Bacciotti \& Eislöffel 1999; Nisini et al. 2005; Podio et al. 2006) an estimate of $\dot{M}_{\text {jet }}$ can be inferred from the determined total density, $n_{\mathrm{H}}$, and the measured gas velocity, $V_{\text {jet }}$, and jet radius, $r_{\text {jet }}$, as $\dot{M}_{\text {jet }}=\mu m_{\mathrm{H}} n_{\mathrm{H}} \pi r_{\text {jet }}^{2} V_{\text {jet }}$, where $\mu=1.49$ is the mean atomic weight and $m_{\mathrm{H}}$ the proton mass. The flux of linear momentum transported by the jet, $\dot{P}_{\text {jet }}$, is then easily obtained as $\dot{P}_{\text {jet }}=\dot{M}_{\text {jet }}$ $V_{\text {jet }}$. As noted in Podio et al. (2006), this way of estimating the mass flux is independent on reddening and distance estimates. On the other hand, it assumes that the jet is uniformly filled at the density derived from the diagnostics within the estimated jet radius, $r_{\text {jet }}$, thus providing basically an upper limit to $\dot{M}_{\text {jet }}$. Such an effect is partially compensated for by regions at higher densities in the jet than those traced by the [S II] lines, which are not taken into account in the calculations (Nisini et al. 2005; Podio et al. 2006).

We separately estimate $\dot{M}_{\text {jet }}$ in the low- and high-velocity components in the two jet lobes. The absolute jet velocity, $V_{\text {jet }}$, is derived from the radial velocities estimated by Gaussian fitting of the [S II] 66731 line profile (see Fig. 4) and correcting for the inclination angle of $65^{\circ}$ (Eislöffel \& Mundt 1998). The jet radius, $r_{\text {jet }}$, has been estimated by Mundt et al. (1991) for distances 

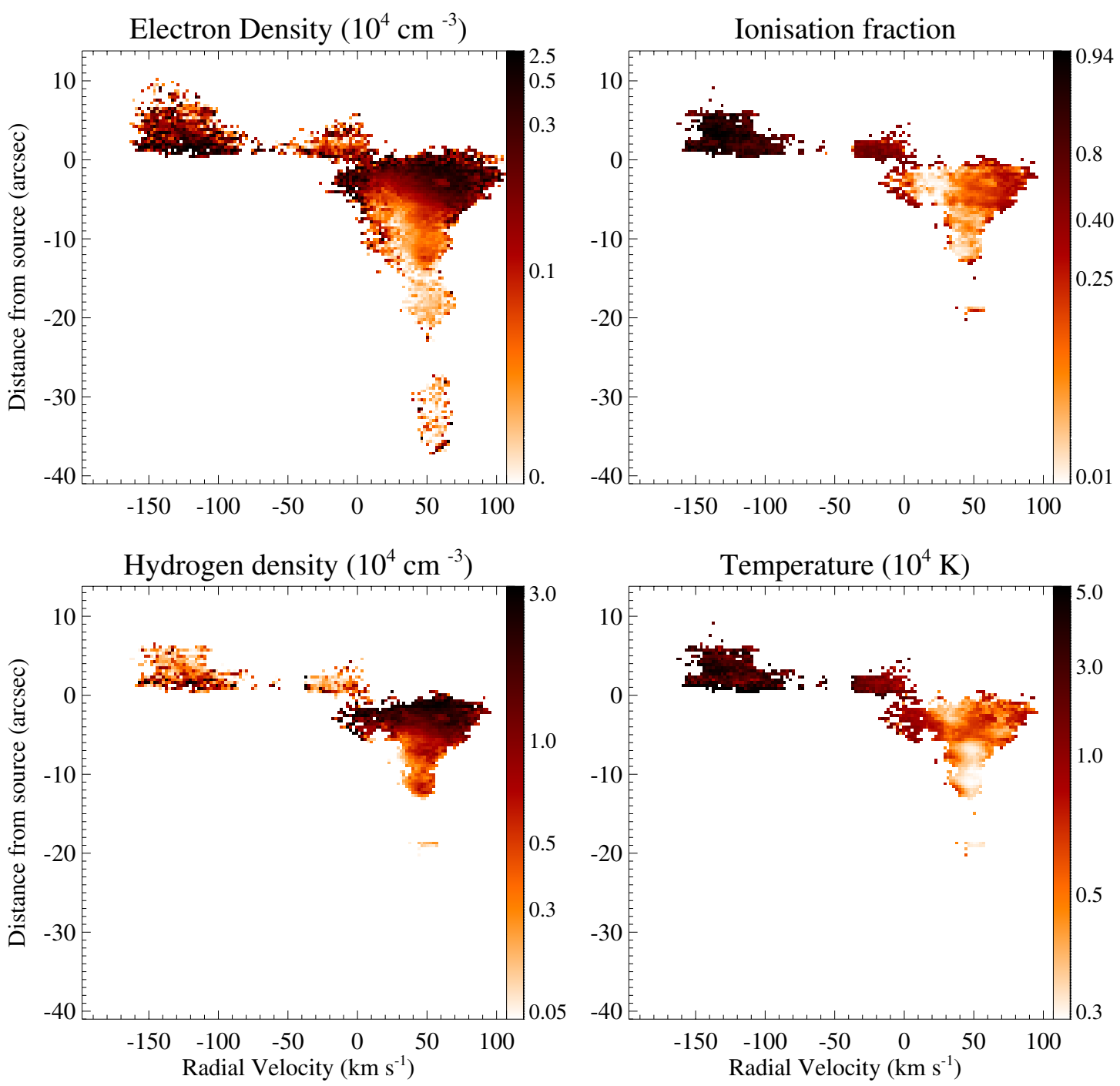

Fig. 6. Position-velocity diagrams of the gas physical conditions in the jet (a histogram equivalent colour scale is used). The electron density, $n_{\mathrm{e}}$, in units of $10^{4} \mathrm{~cm}^{-3}$ (top left), the ionisation fraction, $x_{\mathrm{e}}$ (top right), the temperature, $T_{\mathrm{e}}$, in units of $10^{4} \mathrm{~K}$ (bottom right), and the total density, $n_{\mathrm{H}}$, in units of $10^{4} \mathrm{~cm}^{-3}$ (bottom left) are calculated for each pixel of the spectral images. ( 1 pix $=0.378^{\prime \prime}$ in the spatial direction and $2.15 \mathrm{~km} \mathrm{~s}{ }^{-1}$ in the spectral direction; angular resolution: $F W H M_{\text {seeing }} \sim 0.8^{\prime \prime} \sim 2$ pixels, spectral resolution: $F W H M_{\text {inst }} \sim 8.8 \mathrm{~km} \mathrm{~s}^{-1} \sim 4$ pixels). The errors on the estimated values of $n_{\mathrm{e}}, x_{\mathrm{e}}, T_{\mathrm{e}}, n_{\mathrm{H}}$ may vary between a few percent and $50 \%$ depending on the line signal-to-noise on each pixel.

larger than $\sim 2^{\prime \prime}$ and $\sim 3^{\prime \prime} .5$ in the blue and red lobe, respectively, and by Stapelfeldt et al. (1997) for $d \sim 00^{\prime} 1-5^{\prime \prime}$. The variation of $r_{\text {jet }}$ with distance from the source, obtained by interpolating the estimates from these authors, is shown in the upper panel of Fig. 8 . The errors on the inferred values of $\dot{M}_{\text {jet }}$ are obtained by propagating the errors affecting our estimates of the jet total density, $n_{\mathrm{H}}$, and of the radial velocity, $V_{\mathrm{rad}}$. One should keep in mind, however, that the main source of uncertainty is the jet radius. For example, Mundt et al. (1991) estimated $r_{\text {jet }}$ by applying onedimensional deconvolution method to seeing-limited images of the jet in the $\left[\mathrm{S}_{\mathrm{II}}\right]$ lines and the uncertainty on the obtained values can be as high as $30 \%$. The values of $r_{\text {jet }}$ obtained by Stapelfeldt et al. (1997) through HST/WFPC2 broadband imaging are more accurate because the jet width is resolved thanks to the high angular resolution offered by HST ( 0.' 1$)$. In both cases, however, there is no spectral information, thus estimates of $r_{\text {jet }}$ relative to the different velocity components are not available. Here we assume that the values derived from the works cited above are valid for all velocities, even though Bacciotti et al. (2000) and Lavalley-Fouquet et al. (2000) showed that for the jet from DG Tau, the high-velocity gas is more collimated than the low-velocity component.

The estimated values of $\dot{M}_{\text {jet }}$ in the HVC and in the LVC of the red and the blue lobe are plotted in Fig. 8. In the red lobe $\dot{M}_{\text {jet }}$ turns out to be $\sim 2-6 \times 10^{-9}, \sim 2-3 \times 10^{-9} M_{\odot} \mathrm{yr}^{-1}$ in the high- and low-velocity components, respectively. In the blue lobe $\dot{M}_{\text {jet }}(\mathrm{HVC}) \sim 0.2-2 \times 10^{-8} M_{\odot} \mathrm{yr}^{-1}$ and $\dot{M}_{\text {jet }}(\mathrm{LVC}) \sim 0.3-$ $4 \times 10^{-10} M_{\odot} \mathrm{yr}^{-1}$.

Concerning the linear momentum, in the red lobe $\dot{P}_{\text {jet }}$ turns out to be $\sim 3-9 \times 10^{-7}$ and $\sim 1-2 \times 10^{-7} M_{\odot} \mathrm{yr}^{-1} \mathrm{~km} \mathrm{~s}^{-1}$ in the high- and low-velocity components, respectively, while in the blue lobe it is $\sim 2-6 \times 10^{-6}$ and $\sim 0.05-5 \times 10^{-9} M_{\odot} \mathrm{yr}^{-1} \mathrm{~km} \mathrm{~s}^{-1}$ in the HVC and LVC, respectively. The values of $\dot{M}_{\text {jet }}$ and $\dot{P}_{\text {jet }}$ for the blue lobe LVC are 2-3 orders of magnitude lower than in the HVC, because of the very low velocity (only a few $\mathrm{km} \mathrm{s}^{-1}$ ) coupled to very low total densities $\left(n_{\mathrm{H}} \leq 5 \times 10^{3} \mathrm{~cm}^{-3}\right)$, and are not reported in Fig. 8. In general, the much lower and higher velocities of the LVC and the HVC in the blue lobe with respect to the red one suggest less interaction with the ambient medium, as explained in more detail in Sect. 4.3. 


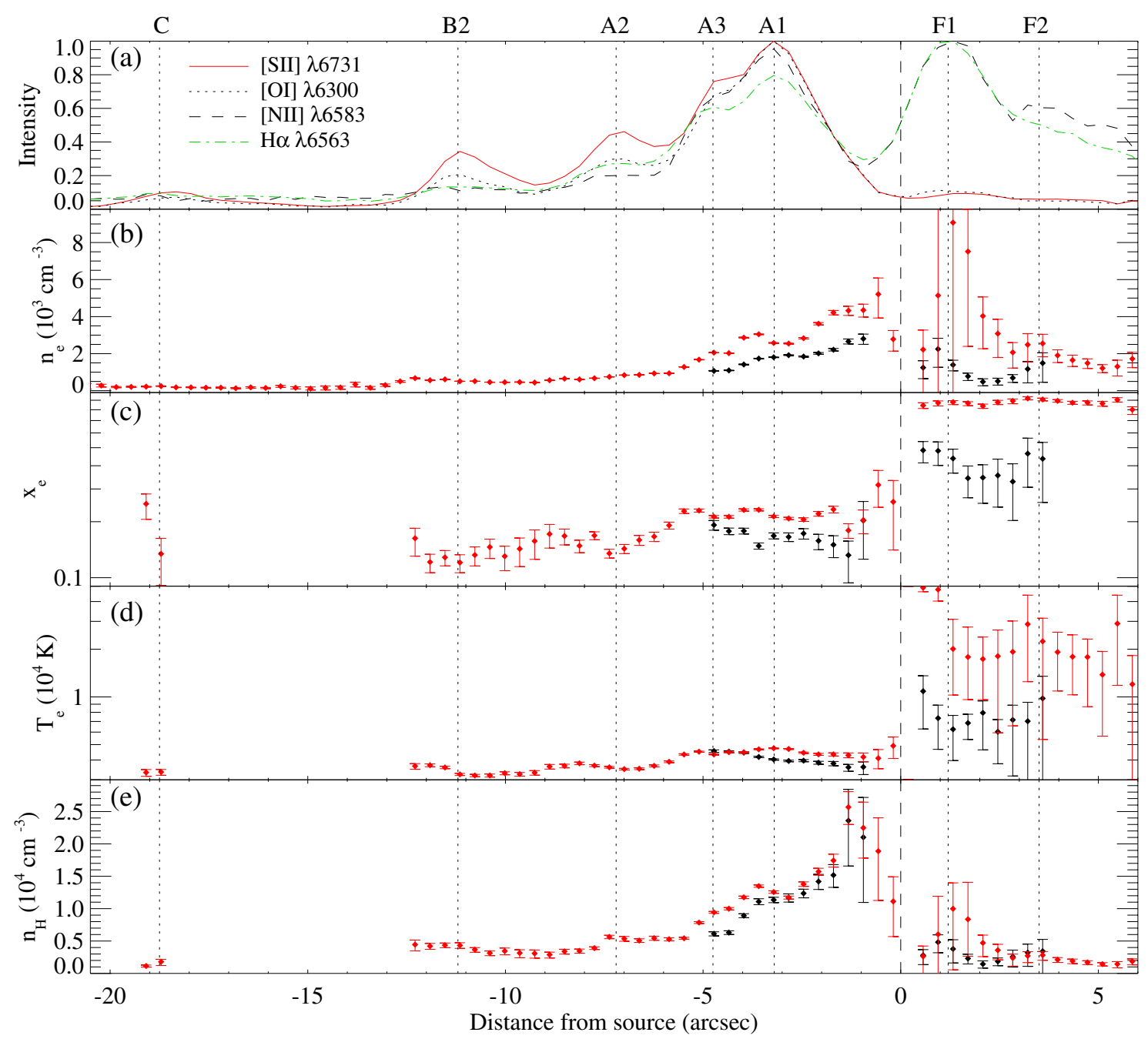

Fig. 7. Variations of the gas physical conditions along the jet and in the low- (LVC, black diamonds) and the high- (HVC, red diamonds) velocity components. The points are a median of the values shown in Fig. 6 over the HVC and the LVC. From top to bottom: a) intensity profiles of the

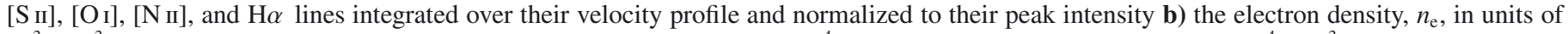
$\left.10^{3} \mathrm{~cm}^{-3} ; \mathbf{c}\right)$ the ionisation fraction, $\left.x_{\mathrm{e}} ; \mathbf{d}\right)$ the temperature, $T_{\mathrm{e}}$, in units of $\left.10^{4} \mathrm{~K} ; \mathbf{e}\right)$ the total density, $n_{\mathrm{H}}$, in units of $10^{4} \mathrm{~cm}^{-3}$.

The average of $\dot{M}_{\text {jet }}$ and $\dot{P}_{\text {jet }}$ over the first $5^{\prime \prime}$ in the highand the low-velocity components of both lobes are summarised in Table 1 . The average values of $\dot{M}_{\text {jet }}$ indicate that summing the HVC and LVC contributions, the mass outflow rate transported by the blue lobe $\left(\dot{M}_{\text {jet }}\right.$ (blue $\left.) \sim 8.2 \times 10^{-9} M_{\odot} \mathrm{yr}^{-1}\right)$ is only $\sim 1.3$ times higher than that transported by the red one $\left(\dot{M}_{\text {jet }}(\right.$ red $)$ $\left.\sim 6.4 \times 10^{-9} M_{\odot} \mathrm{yr}^{-1}\right)$. Considering the large error on this quantity, it can be said that in practice the amount of mass transported by the jet in the two lobes is comparable, despite the large differences in density and velocity. This situation, already found for the asymmetric bipolar jet from the T Tauri star RW Aur by Melnikov et al. (2009), indicates that the ejection power is similar on the two sides of the disc, as it is further discussed in Sect. 4.3.

The rate of mass ejected into the jet, $\dot{M}_{\text {jet }}$, cannot be compared with the mass accretion rate onto the star, $\dot{M}_{\text {acc }}$, because DG Tau B is obscured by the optically thick accretion disc, which prevents veiling measurements at optical wavelengths (e.g., Hartigan et al. 1995). The inferred value of $\sim 6-$ $8 \times 10^{-9} M_{\odot} \mathrm{yr}^{-1}$ is on the same order of magnitude as those estimated for more evolved T Tauri stars $\left(\dot{M}_{\text {jet }}\right.$ (TTSs) $\sim 10^{-10}-5 \times$ $10^{-8} M_{\odot} \mathrm{yr}^{-1}$ ) through the same method as adopted in this paper
(Coffey et al. 2008; Melnikov et al. 2009; Maurri et al., in prep.) or also from the luminosity of optical forbidden lines (Hartigan et al. 1995), while it turns out to be lower than the typical values inferred for $\mathrm{HH}$ jets emitted by young Class 0/I sources $\left(\dot{M}_{\text {jet }}\right.$ (Class 0/I) $7 \times 10^{-8}-4 \times 10^{-7} M_{\odot} \mathrm{yr}^{-1}$, Hartigan et al. 1994; Bacciotti \& Eislöffel 1999; Podio et al. 2006). The derived low values of $\dot{M}_{\text {jet }}$ may indicate that the bulk of the gas is emitting in lower excitation lines lying at far-infrared wavelengths, such as the [OI] $63 \mu \mathrm{m}$ line. This hypothesis seems to be confirmed by preliminary results from the analysis of Herschel/PACS observations (Podio et al., in prep.).

The linear momentum transported by the blue lobe ( $\dot{P}_{\text {jet }}$ (blue) $\sim 2.5 \times 10^{-6} M_{\odot} \mathrm{yr}^{-1} \mathrm{~km} \mathrm{~s}^{-1}$ ) is $\sim 3$ times higher than in the red one $\left(\dot{P}_{\text {jet }}(\right.$ red $\left.) \sim 8 \times 10^{-7} M_{\odot} \mathrm{yr}^{-1} \mathrm{~km} \mathrm{~s}^{-1}\right)$, owing to the multiplication by the jet velocity. In order to understand the dynamical relationship between the jet and coaxial molecular outflows, it is very interesting to compare the estimated value of the momentum flux transported by the jet, with the momentum flux transported by the surrounding molecular outflow detected in CO lines. Mitchell et al. (1994) estimated a momentum flux of $\sim 1.9 \times 10^{-6} M_{\odot} \mathrm{yr}^{-1} \mathrm{~km} \mathrm{~s}^{-1}$ by assuming a distance of $\sim 160 \mathrm{pc}$ and an inclination angle with respect to the sky of $\sim 15^{\circ}$. Correcting this value for recent more 


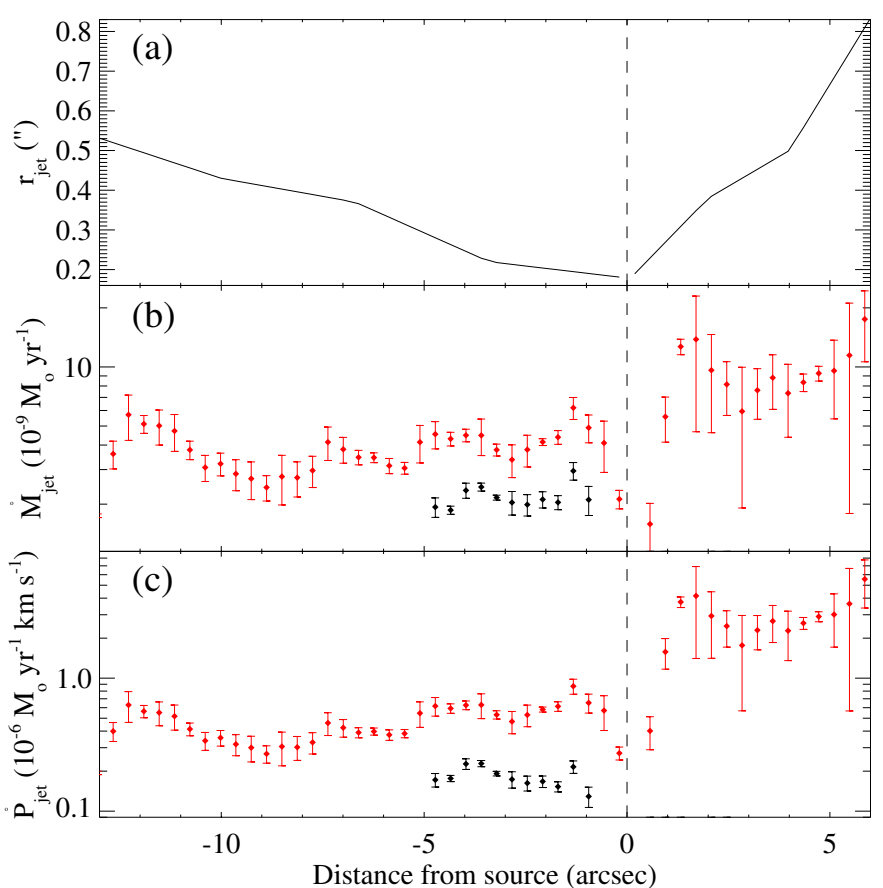

Fig. 8. From top to bottom: a) jet radius, $r_{\text {jet }}$, in arcseconds (as estimated by Mundt et al. (1991) for distances larger than $\sim 2^{\prime \prime}$ and $\sim 3.5^{\prime \prime}$ in the blue and red lobe, respectively, and by Stapelfeldt et al. (1997) for lower distances); (b), c)) mass flux rate, $\dot{M}_{\text {jet }}$, in units of $10^{-9} M_{\odot} \mathrm{yr}^{-1}$, and flux of linear momentum, $\dot{P}_{\text {jet }}$, in units of $10^{-6} M_{\odot} \mathrm{yr}^{-1} \mathrm{~km} \mathrm{~s}^{-1}$, estimated in the high- (HVC, red points) and low- (LVC, black points) velocity components.

accurate estimates of the distance $(d \sim 140 \mathrm{pc})$ and of the inclination angle $\left(i \sim 25^{\circ}\right.$, Eislöffel \& Mundt 1998), one obtains $\dot{P}_{\text {outflow }} \leq 10^{-6} M_{\odot} \mathrm{yr}^{-1} \mathrm{~km} \mathrm{~s}^{-1}$. This value is comparable to the momentum estimated for the jet, indicating that the latter is powerful enough to drive the molecular outflow detected at millimeter wavelengths. Precisely how the jet transfers its linear momentum to the surrounding medium, however, remains an open question (see, e.g., Downes \& Cabrit 2007).

\subsection{Dust reprocessing along the jet: investigating the $\mathrm{Ca}, \mathrm{Ni}$, and $\mathrm{Fe}$ gas-phase abundance}

The KECK spectra of DG Tau B include also a number of emission lines from "refractory" species such as $\mathrm{Fe}, \mathrm{Ni}$, and $\mathrm{Ca}$. These species are known to be highly depleted in the interstellar medium because their atoms may be locked onto dust grains (Savage \& Sembach 1996). Estimates of the Ca, Fe, and Ni gasphase abundance in Orion and $\zeta$ Oph indicate that they are depleted up to four orders of magnitude with respect to their solar abundances (Baldwin et al. 1991; Savage \& Sembach 1996; Esteban et al. 2004). On the other hand, dust grains can be partially or completely destroyed in the jet launching region as well as in the shocks occurring along the flow, releasing $\mathrm{Ca}, \mathrm{Fe}$, and $\mathrm{Ni}$ atoms into the gas-phase.

The models (Isella \& Natta 2005) and also the near-infrared interferometric observations (Akeson et al. 2005; Eisner et al. 2007) show that the inner part of the accretion disc around $T$ Tauri and Herbig Ae/Be stars is dust-free, because dust grains are destroyed by the stellar radiation up to the so-called dust evaporation radius, $R_{\text {evp }}$. This is located at $\sim 0.1-1.5 \mathrm{AU}$ in Herbig stars (Eisner et al. 2007) and at $\sim 0.05-0.3 \mathrm{AU}$ in T Tauri stars (Akeson et al. 2005). For younger Class I sources there are no direct estimates of $R_{\text {evp }}$ because of their embedded nature. However, we can obtain a rough estimate of the position of $R_{\text {evp }}$ for DG Tau B, assuming that the model by Isella \& Natta (2005) is still valid for a source this young. For a luminosity of $\sim 0.7-0.9 L_{\odot}$ (Jones \& Cohen 1986; Watson et al. 2004) and a mass accretion rate of at least $\sim 10$ times higher than the inferred $\dot{M}_{\text {jet }}$ we find that $R_{\text {evp }}$ is located at about 1-6 times the position of the truncation radius, $R_{\mathrm{t}}$, where the stellar magnetosphere truncates the disc. (The position of $\mathrm{R}_{t}$ is computed from Eq. (2.2a,b) of Shu et al. (1994), assuming typical temperature and mass for DG Tau B spectral type ( $\leq \mathrm{K} 6$, Watson et al. 2004), $T \sim 3000-5000 \mathrm{~K}$ and $M \sim 0.1-$ $1 M_{\odot}$, and a stellar magnetic field strength, $\mathrm{B}_{*}$, of $\sim 0.5-3 \mathrm{kG}$, Johns-Krull 2007). Thus, if the jet originates in the inner region of the disc $\left(r<R_{\mathrm{evp}}\right)$, we expect $\mathrm{Ca}$, $\mathrm{Fe}$, and $\mathrm{Ni}$ to be present in solar abundance. On the contrary, if the jet originates from a region of the disc extending beyond $R_{\text {evp }}$, part of the dust is lifted with the jet material, and these species will be observed to be depleted in the jet with respect to their solar abundances.

The shocks occurring along the jet can also affect the gasphase abundances of refractory species. Theoretical models of C- and J-shocks showed that processes such as thermal and inertial sputtering, photoevaporation, and shattering can destroy from a few percent up to $\sim 90 \%$ of the dust grains, depending on a number of parameters such as the shock velocity, the gas preshock density, the intensity of the magnetic field, and the size and structure of the dust grains (e.g., Jones et al. 1994; Jones 2000; May et al. 2000; Draine 2004; Guillet et al. 2009).

Thus, we can observationally test the predictions of jet launching models and dust reprocessing in shocks by investigating the gas-phase abundances of $\mathrm{Ca}, \mathrm{Fe}$, and $\mathrm{Ni}$ along jets.

To this aim we analyse the spatio-kinematical distribution of the emission from $\mathrm{Ca}, \mathrm{Fe}$, and $\mathrm{Ni}$ in the jet, and we estimate the gas-phase abundance of calcium, $[\mathrm{Ca} / \mathrm{H}]_{\text {gas }}$ (hereafter $[\mathrm{Ca}]_{\text {gas }}$ ). The PV diagrams in Fig. 2 show that the emission in these lines is confined to the high velocities in the red and in the blue lobe. The velocity profiles suggest that the atoms of $\mathrm{Ca}, \mathrm{Fe}$, and $\mathrm{Ni}$, which are highly depleted in the ISM, may be released in the gas-phase in the inner disc region and/or in the shocks. In both cases higher abundances are expected in the high-velocity component. Indeed, on the one hand, the jet launch models predict that the gas extracted from the inner disc region is accelerated to higher velocities with respect to the gas extracted from the outer disc. On the other hand, the destruction rate in a shock is highly dependent on the shock velocity. Thus a higher destruction rate is expected in the strongest shocks and at high velocities.

We estimate the $\mathrm{Ca}$ gas-phase abundance following the procedure illustrated in Nisini et al. (2005) and Podio et al. (2006, 2009), which consists of comparing observed and predicted ratios between refractory and non-refractory species such as sulphur, which are expected to be present in solar abundance in the interstellar medium. Following these studies we compare observed [Ca II] $\lambda 7291 /\left[\mathrm{S}_{\text {II }}\right] \lambda 6731$ ratios (hereafter $[\mathrm{Ca} I \mathrm{II}] /[\mathrm{S}$ II] $)$, with those computed through the estimated parameters $\left(n_{\mathrm{e}}, x_{\mathrm{e}}\right.$, and $\left.T_{\mathrm{e}}\right)$ and assuming solar abundance for $\mathrm{Ca}$ and S (Asplund et al. 2005). The abundance of calcium in gas-phase in the jet with respect to its solar value is given by the ratio between observed and predicted $[\mathrm{Ca} I] /[\mathrm{S}$ II] ratios: $[\mathrm{Ca}]_{\text {gas }} /[\mathrm{Ca}]_{\text {solar }}=\left([\mathrm{Ca} \text { II }] /\left[\mathrm{S}_{\mathrm{II}}\right]\right)_{\text {obs }} /([\mathrm{Ca} \text { II }] /[\mathrm{S} \text { II }])_{\text {predicted }}$. Following Hartigan et al. (2004) we assume that the [Ca II] lines are collisionally excited and compute the level populations. As in previous works (Nisini et al. 2005; Podio et al. 2006, 2009), we also assume that there is no calcium in the form of $\mathrm{Ca}^{0}$, since its ionisation potential is very low, $\sim 6.1 \mathrm{eV}$. (Note that emission in the $\mathrm{Na}$ I $\mathrm{D}$ lines is barely detected only at the peak velocity of knots A1, A3 at $\sim 6 \sigma$ ). Moreover, we assume that at every 


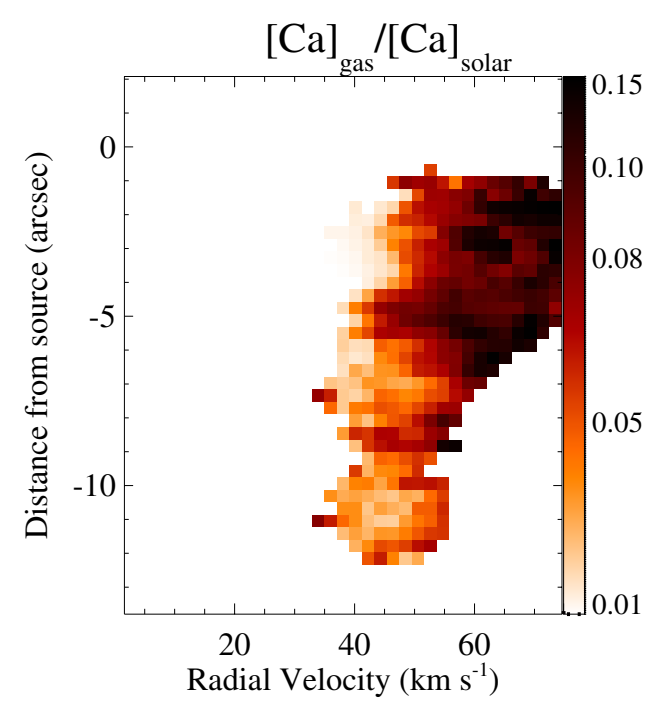

Fig. 9. Position-velocity diagram of the Ca gas-phase abundance estimated as the ratio between observed and predicted (by assuming Ca solar abundance) $[\mathrm{Ca}$ II $] \lambda 7291 /[\mathrm{S}$ II] $] 6731$ ratios.

position along the jet, the amount of calcium ionised twice with respect to singly ionised calcium, i.e. $\mathrm{Ca}^{++} / \mathrm{Ca}^{+}$, is equal to the hydrogen ionisation fraction, $x_{\mathrm{e}}$. As explained in Podio et al. (2009), the latter assumption is justified because the ionisation potential of $\mathrm{Ca}^{+}$is similar to that of hydrogen $(\sim 11.9 \mathrm{eV}$ and $\sim 13.6 \mathrm{eV}$, respectively), as are the recombination and collisional ionisation coefficients for temperatures lower than $3 \times 10^{4} \mathrm{~K}$. Thus, $\mathrm{Ca}^{+}$and $\mathrm{H}^{0}$ atoms can be thought of as undergoing the same processes when moving along the jet.

We have no estimates of the visual extinction, therefore we cannot correct the $\mathrm{Ca}^{+}$and $\mathrm{S}^{+}$line fluxes for reddening. This implies that the observed ratios (assuming $A_{\mathrm{V}}=0$ ) are actually upper limits to the real values (which decrease for increasing $\left.A_{\mathrm{V}}\right)$. Thus, the calcium depletion inferred from our analysis is a lower limit.

Even so, the results shown in Fig. 9 illustrate that calcium turns out to be strongly depleted, its gas-phase abundance varying between a few percent and $15 \%$ of its solar value $\left([\mathrm{Ca} / \mathrm{H}]_{\text {solar }} \sim 2.04 \times 10^{-6}\right.$, Asplund et al. 2005). Interestingly, the $\mathrm{Ca}$ abundance is at a maximum close to the source and at high velocities, where the broad velocity profiles and high values of $n_{\mathrm{e}}$ and $x_{\mathrm{e}}$ suggest the presence of strong shocks. We also derived an estimate of the Ca gas-phase abundance in the blue lobe, despite the very low signal-to-noise of calcium emission here. It turns out that the observed $[\mathrm{Ca}$ II $] /\left[\mathrm{S}_{\mathrm{II}}\right]$ ratio is $\sim 1-3$ times the predicted one, implying that $\mathrm{Ca}$ is completely released into the gas-phase in the blue lobe, where the very high excitation conditions $\left(x_{\mathrm{e}}, T_{\mathrm{e}}\right)$ indicate very high shock velocities. Similar results have also been found in the HH 111 protostellar jet (Podio et al. 2009) and confirm the dependence of elemental depletion on shock velocity as predicted by shock dust destruction models (e.g., Guillet et al. 2009).

\subsection{Investigating the origin of velocity components and asymmetries in stellar jets}

The presence of a low- and a high-velocity component in jets from young stars was first noted by Hamann (1994). Hirth et al. (1997) analysed [S II], [O I], and [N II] forbidden emission lines in 38 TTSs and report $58 \%$ of them showing a LVC and a HVC, with the LVC being always less extended than the HVC. Similarly, two velocity components have been detected at the base of $\mathrm{HH}$ jets emitted by Class 0/I sources and in these cases, the LVC is also less extended than the HVC. However, while in the flow from T Tauri stars the LVC is confined within 20-200 AU (Hirth et al. 1997), the jets emitted by younger Class 0/I sources present the LVC extending up to 1000-2000 AU from the source (Garcia Lopez et al. 2008, 2010). In agreement with these results the LVC from DG Tau B is observed up to 500-700 AU from the source.

Moreover, Hirth et al. (1997) note that the LVC is not observed in the $[\mathrm{N}$ II] lines, suggesting that the LVC is less excited than the HVC, i.e. $\mathrm{N}$ is ionised only once in the low-velocity gas. More recent deeper observations, however, have shown low velocity [N II] emission in a few objects, such as DG Tau and CW Tau, allowing us to apply spectral diagnostic techniques and to infer the physical properties of the gas in the two velocity components. Therefore, we compare our results with those found for DG Tau and CW Tau to investigate the properties and the origin of the high- and the low-velocity components in jets.

The values of the physical parameters summarised in Table 1 show that in the jet from DG Tau B the HVC is denser and more excited than the LVC in the blue and the red lobe. Higher electron densities and ionisation fractions in the HVC have been found in DG Tau (Lavalley-Fouquet et al. 2000; Maurri et al., in prep.), and in CW Tau (Coffey et al. 2008) as well. On the other hand, the analysis of the line ratios in a large sample of TTSs by Hamann (1994) suggests higher temperatures but lower electron densities in the HVC. Combining the results from DG Tau B with these previous estimates, we can conclude that there is clear evidence that the HVC is more excited than the LVC. To understand if the HVC is also denser is more difficult, because in many cases an accurate estimate of the total density is limited because the [S II] lines used to infer $n_{\mathrm{e}}$ are saturated in one or both velocity components and therefore one only obtains lower limits to the real density (Lavalley-Fouquet et al. 2000; Coffey et al. 2008; Maurri et al., in prep.). For jet from Class 0/I sources, the electron density estimated through near-infrared [Fe II] lines is higher at high velocities along $\mathrm{HH} 1$ and at low velocities in $\mathrm{HH} 34$ and $\mathrm{HH}$ 46-47. Because no estimate of the ionisation fraction is available, however, it is not possible to understand which is the denser component. Interestingly, higher densities and excitation conditions at high velocities have been noted at the position of the shock surfaces detected along the HH 111 jet $20^{\prime \prime}-60^{\prime \prime}$ away from the source (Podio et al. 2009).

That the LVC shows lower excitation conditions suggests that this component originates in an extended disc-wind where the gas extracted from an outer region of the disc with respect to that emitting the HVC is accelerated at lower velocities and gives rise to slower shocks (hence, the lower ionisation fraction and temperature). This hypothesis seems to be confirmed by high angular resolution observations of DG Tau obtained with an adaptive optics system and/or with the Hubble space telescope, which resolve the jet perpendicular to its axis and show that the HV gas is more collimated than the LV gas (Bacciotti et al. 2000; Lavalley-Fouquet et al. 2000; Maurri et al., in prep.).

Another interesting and still unexplained characteristic of jets from young stars is that they are often asymmetric. Hirth et al. (1994) noted that 8 out of 15 objects for which estimates of the radial velocities in both lobes are available show a velocity asymmetry with the ratio between the velocity of the fast lobe and the velocity of the slow lobe, $V_{\mathrm{f}} / V_{\mathrm{s}}$, ranging between 1.4 and 2.6. However, a statistical study of asymmetric jets, aimed to investigate the origin of this asymmetry, is hampered because 
in most of the cases one lobe is much more extincted or intrinsically fainter than the other, which makes it difficult to derive the gas properties in both lobes. Our deep KECK/HIRES observations allow us for the first time to detect both lobes of DG Tau B in all emission lines, which are employed in our diagnostic techniques, and thus to derive $n_{\mathrm{e}}, x_{\mathrm{e}}, T_{\mathrm{e}}$, and $n_{\mathrm{H}}$ in both lobes.

We compare our results with those found by Melnikov et al. (2009) for the well-known asymmetric jet from RW Aur, and find that the jets from the two stars have very similar kinematical and physical characteristics. First of all, in both cases the blue lobes are unusually much fainter than the red ones. We note that in both cases the density of the blue lobe is lower, implying intrinsically fainter emission. The blue lobe is also faster in both targets, probably indicating less interaction with the environment on that side of the system. In more detail, the blue lobe is $\sim 1.8$ times faster than the red one in RW Aur and $\sim 2.3$ in DG Tau B, it is more ionised by a factor of $\sim 3$ in RW Aur and $\sim 3.3$ in DG Tau B, and in both jets the fast lobe is less dense than the slow one by a factor $\sim 0.3$. An important difference between the two jets is that while the width of the jet from RW Aur is similar in the two lobes $\left(r_{\text {jet } \mathrm{f}} / r_{\text {jet s }} \sim 1\right)$, the available images of DG Tau B indicate that the fast lobe is less collimated than the slow one $\left(r_{\text {jet f }} / r_{\text {jet s }} \sim 2\right)$. The most interesting similarity, however, is that in both DG Tau B and RW Aur the mass transported by the two lobes is comparable (with a ratio of about 1.3 in both targets, smaller than the error on the estimates) despite the observed asymmetry in the physical quantities. Finally, the flux of linear momentum transported by the fast lobe is obviously higher than that transported by the slow one in both systems $\left(\dot{P}_{\text {jet f }} / \dot{P}_{\text {jet s }} \sim 1.4\right.$ for RW Aur and $\sim 3$ for DG Tau B $)$. We note that similar $\dot{M}_{\text {jet }}$ in the two jet lobes has also been found in other stellar jets showing asymmetries in the physical conditions as, e.g., the HH 30 jet (Bacciotti et al. 1999) and the bipolar jet from the Herbig Ae/Be star LkH $\alpha 233$ (Melnikov et al. 2008).

The striking similarity between the properties of these asymmetric jets suggests that similar effects are at work. As discussed in Melnikov et al. (2009), the fact that the global mass outflow rate is comparable on the two sides of the DG Tau B system appears to indicate that as in the RW Aur case, the power transferred by the rotating disc is the same on both sides of the disc, supporting the validity of a magneto-centrifugal mechanism. In this scenario, however, an asymmetric ambient medium can lead to an asymmetric interaction of the disc with the environment, hence different mass load, lever arms and/or radial extensions of the disc region that produces the jet on the two sides (Ferreira et al. 2006). For example, if the ambient radiation field is stronger on one side, this increases the level of ionisation on the surface of the disc on that side, leading to enhanced mass load on the magnetic field lines, and/or to a larger jet launching region. For DG Tau B, a larger mass-load and interaction with the surrounding medium in the red lobe is suggested by the detection of a redshifted molecular outflow in the millimeter CO lines (Mitchell et al. 1994).

\section{Summary and conclusions}

We investigated the kinematical and physical structure of the HH 159 jet emitted by the young Class I source DG Tau B by means of high spectral resolution observations acquired with KECK/HIRES. Contrary to previous studies (e.g., Eislöffel \& Mundt 1998), we couple the analysis of the jet kinematics with a detailed study of its physical and dynamical properties in each of its lobes and velocity components. Our method involves the analysis of selected line ratios through the so-called BE diagnostic technique (Bacciotti \& Eislöffel 1999) to infer the gas physical conditions $\left(n_{\mathrm{e}}, x_{\mathrm{e}}, T_{\mathrm{e}}\right.$, and $\left.n_{\mathrm{H}}\right)$. Our main conclusions are:

- The velocity variations along the jet indicates that the ejection direction is changing because of precession and/or interaction with the surrounding medium. The electron and total density as well as the ionisation fraction and the temperature are decreasing along both lobes because of gas dilution, i.e. jet propagation in a conical geometry, and/or stronger shocks at the jet base, where the ejected material interacts with the dense parental cloud.

- The lines show a complex velocity and excitation structure with multiple velocity components. The high-velocity component, HVC, is more extended than the low-velocity component, LVC, which is fading at 500-700 AU from the source, similarly to what was previously observed for other jets from Class 0/I sources (Garcia Lopez et al. 2008) and, on smaller scales, for jets from CTTSs (e.g., Hirth et al. 1997). The HVC appears to be denser and more excited, suggesting that the LV gas is extracted from an outer region of the disc with respect to the HV gas and is therefore accelerated at lower velocities.

- The jet shows a strong asymmetry: the blue lobe is faster and more excited than the red lobe, but less dense and collimated, suggesting that the interaction of the ejected material with the ambient medium is stronger on the red-lobe side. This asymmetry is similar to the one observed in other jets, and in particular in RW Aur (Melnikov et al. 2009).

- Despite the observed asymmetries, the mass loss rate is similar in the two lobes $\left(\dot{M}_{\text {jet }} \sim 6-8 \times 10^{-9} M_{\odot} \mathrm{yr}^{-1}\right)$, as in other asymmetric jets. This means that the the power transferred by the rotating disc is the same on both sides of the system, as expected if the jet is launched by a magneto-centrifugal engine. The observed asymmetries can be explained in the framework of an MHD disc wind, if the latter propagates in an inhomogeneous ambient medium (Ferreira et al. 2006).

- The redshifted molecular outflow detected in the CO millimeter lines (Mitchell et al. 1994) supports the idea of an asymmetric ambient medium and a stronger jet-ISM interaction on the red-lobe side. The flux of linear momentum transported by the jet is comparable to that estimated for the molecular outflow, therefore the latter can be jet-driven $\left(\dot{P}_{\text {jet }} \sim 0.8-\right.$ $2.5 \times 10^{-6} M_{\odot} \mathrm{yr}^{-1} \mathrm{~km} \mathrm{~s}^{-1}$ ).

- The depletion of the Ca gas-phase abundance with respect to its solar abundance $\left([\mathrm{Ca}]_{\text {gas }} /[\mathrm{Ca}]_{\text {solar }} \sim 0.01-0.15\right)$ indicates that most $\mathrm{Ca}$ atoms are locked onto dust grains. The minimum depletion is observed close to the source and in the HVC, where the strongest shocks are occurring, in agreement with the predictions from models of dust reprocessing in shocks (e.g., Guillet et al. 2009). The presence of dust in the jet may imply that the material is extracted from a region of the disc that extends beyond the so-called dust evaporation radius, $R_{\text {evp }}$. However, more stringent constraints on the jet launching region can only be derived by analysing higher angular resolution observations, to infer the Ca gas-phase abundance in the first tens of AU from the source where the jet material has not been reprocessed by shocks, and for more unembedded sources, for which the location of $R_{\text {evp }}$ can be derived from near-infrared interferometric observations (e.g., Akeson et al. 2005).

Acknowledgements. The data presented in this paper were obtained at the W. M. Keck Observatory, which is operated as a scientific partnership among the 
California Institute of Technology, the University of California, and the National Aeronautics and Space Administration. The Observatory was made possible by the generous financial support of the W. M. Keck Foundation. The authors wish to recognize and acknowledge the very significant cultural role and reverence that the summit of Mauna Kea has always had within the indigenous Hawaiian community. We are most fortunate to have the opportunity to conduct observations from this mountain. J.E. thanks the Institute for Astronomy, UH, for its hospitality during his stay as an Otto-Hahn fellow of the Max-Planck Society. L.P. thanks the Irish Research Council for Science, Engineering and Technology, which partially funded her work. Finally, this work was partially supported by the European Community's Marie Curie Research and Training Network JETSET (Jet Simulations, Experiments and Theory) under contract MRTN-CT-2004-005592.

\section{References}

Akeson, R. L., Walker, C. H., Wood, K., et al. 2005, ApJ, 622, 440

Asplund, M., Grevesse, N., \& Sauval, A. J. 2005, Cosmic Abundances as Records of Stellar Evolution and Nucleosynthesis, 336, 25

Bacciotti, F., \& Eislöffel, J. 1999, A\&A, 342, 717

Bacciotti, F., Eislöffel, J., \& Ray, T. P. 1999, A\&A, 350, 917

Bacciotti, F., Mundt, R., Ray, T. P., et al. 2000, ApJ, 537, L49

Baldwin, J. A., Ferland, G. J., Martin, P. G., et al. 1991, ApJ, 374, 580

Beck-Winchatz, B., Böhm, K. H., \& Noriega-Crespo, A. 1994, PASP, 106, 1271

Beck-Winchatz, B., Böhm, K.-H., \& Noriega-Crespo, A. 1996, AJ, 111, 346

Böhm, K.-H., \& Matt, S. 2001, PASP, 113, 158

Cabrit, S., Codella, C., Gueth, F., et al. 2007, A\&A, 468, L29

Caratti o Garatti, A., Froebrich, D., Eislöffel, J., Giannini, T., \& Nisini, B. 2008, A\&A, 485, 137

Coffey, D., Bacciotti, F., \& Podio, L. 2008, ApJ, 689, 1112

Davis, C. J., Hodapp, K. W., \& Desroches, L. 2001, A\&A, 377, 285

Downes, T. P., \& Cabrit, S. 2007, A\&A, 471, 873

Draine, B. T. 2004, The Cold Universe, Saas-Free Advanced Course, 32 (Springer-Verlag), 213

Eislöffel, J., \& Mundt, R. 1998, AJ, 115, 1554

Eisner, J. A., Chiang, E. I., Lane, B. F., \& Akeson, R. L. 2007, ApJ, 657, 347

Esteban, C., Peimbert, M., García-Rojas, J., et al. 2004, MNRAS, 355, 229

Ferreira, J., Dougados, C., \& Cabrit, S. 2006, A\&A, 453, 785

Garcia Lopez, R., Nisini, B., Giannini, T., et al. 2008, A\&A, 487, 1019

Garcia Lopez, R., Nisini, B., Eislöffel, J., et al. 2010, A\&A, 511, A5

Giannini, T., Massi, F., Podio, L., et al. 2005, A\&A, 433, 941

Guillet, V., Jones, A. P., \& Pineau Des Forêts, G. 2009, A\&A, 497, 145

Hamann, F. 1994, ApJS, 93, 485

Hartigan, P., \& Morse, J. 2007, ApJ, 660, 426

Hartigan, P., Morse, J. A., \& Raymond, J. 1994, ApJ, 436, 125

Hartigan, P., Edwards, S., \& Ghandour, L. 1995, ApJ, 452, 736

Hartigan, P., Edwards, S., \& Pierson, R. 2004, ApJ, 609, 261
Hirth, G. A., Mundt, R., Solf, J., \& Ray, T. P. 1994, ApJ, 427, L99

Hirth, G. A., Mundt, R., \& Solf, J. 1997, A\&AS, 126, 437

Hodapp, K. W., Bally, J., Eislöffel, J., \& Davis, C. J. 2005, AJ, 129, 1580

Isella, A., \& Natta, A. 2005, A\&A, 438, 899

Johns-Krull, C. M. 2007, ApJ, 664, 975

Jones, A. P. 2000, J. Geophys. Res., 105, 10257

Jones, A. P., Tielens, A. G. G. M., Hollenbach, D. J., \& McKee, C. F. 1994, ApJ, 433, 797

Jones, B. F., \& Cohen, M. 1986, ApJ, 311, L23

Königl, A., \& Pudritz, R. E. 2000, Protostars and Planets IV, 759

Lavalley-Fouquet, C., Cabrit, S., \& Dougados, C. 2000, A\&A, 356, L41

Luhman, K. L., Allen, P. R., Espaillat, C., Hartmann, L., \& Calvet, N. 2010, ApJS, 186, 111

May, P. W., Pineau des Forêts, G., Flower, D. R., et al. 2000, MNRAS, 318, 809

Melnikov, S., Woitas, J., Eislöffel, J., et al. 2008, A\&A, 483, 199

Melnikov, S. Y., Eislöffel, J., Bacciotti, F., Woitas, J., \& Ray, T. P. 2009, A\&A, 506, 763

Mitchell, G. F., Hasegawa, T. I., Dent, W. R. F., \& Matthews, H. E. 1994, ApJ, 436, L177

Mitchell, G. F., Sargent, A. I., \& Mannings, V. 1997, ApJ, 483, L127

Mouri, H., \& Taniguchi, Y. 2000, ApJ, 534, L63

Mundt, R., \& Fried, J. W. 1983, ApJ, 274, L83

Mundt, R., Brugel, E. W., \& Buehrke, T. 1987, ApJ, 319, 275

Mundt, R., Ray, T. P., \& Raga, A. C. 1991, A\&A, 252, 740

Nisini, B., Caratti o Garatti, A., Giannini, T., \& Lorenzetti, D. 2002, A\&A, 393, 1035

Nisini, B., Bacciotti, F., Giannini, T., et al. 2005, A\&A, 441, 159

Nisini, B., Codella, C., Giannini, T., et al. 2007, A\&A, 462, 163

Padgett, D. L., Brandner, W., Stapelfeldt, K. R., et al. 1999, AJ, 117, 1490

Padgett, D. L., Stapelfeldt, K. R., \& Sargent, A. F. 2000, BAAS, 32, 1481

Pesenti, N., Dougados, C., Cabrit, S., et al. 2003, A\&A, 410, 155

Podio, L., Bacciotti, F., Nisini, B., et al. 2006, A\&A, 456, 189

Podio, L., Medves, S., Bacciotti, F., Eislöffel, J., \& Ray, T. 2009, A\&A, 506, 779

Pyo, T.-S., Kobayashi, N., Hayashi, M., et al. 2003, ApJ, 590, 340

Raga, A. C., \& Böhm, K. H. 1986, ApJ, 308, 829

Rodriguez, L. F., Anglada, G., \& Raga, A. 1995, ApJ, 454, L149

Sauty, C., \& Tsinganos, K. 1994, A\&A, 287, 893

Savage, B. D., \& Sembach, K. R. 1996, ARA\&A, 34, 279

Shu, F., Najita, J., Ostriker, E., et al. 1994, ApJ, 429, 781

Shu, F. H., Najita, J. R., Shang, H., \& Li, Z.-Y. 2000, Protostars and Planets IV, 789

Solf, J., Böhm, K. H., \& Raga, A. 1988, ApJ, 334, 229

Stapelfeldt, K., Burrows, C. J., Krist, J. E., \& WFPC2 Science Team 1997, Herbig-Haro Flows and the Birth of Stars, 182, 355

van Dokkum, P. G. 2001, PASP, 113, 1420

Vogt, S. S., Allen, S. L., Bigelow, B. C., et al. 1994, Proc. SPIE, 2198, 362

Watson, D. M., Kemper, F., Calvet, N., et al. 2004, ApJS, 154, 391

Woitas, J., Ray, T. P., Bacciotti, F., Davis, C. J., \& Eislöffel, J. 2002, ApJ, 580, 336 